\title{
Stochastic computational model of 3D acoustic noise predictions for nacelle liners
}

\author{
Vincent Dangla* \\ Airbus Operations S.A.S, 316 Route de Bayonne, 31300 Toulouse, France. \\ Université Gustave Eiffel, MSME UMR 8208 CNRS, 77450 Marne-la-Vallée, France. \\ Christian Soize ${ }^{\dagger}$ \\ Université Gustave Eiffel, MSME UMR 8208 CNRS, 77450 Marne-la-Vallée, France \\ Guilherme Cunha ${ }^{\ddagger}$, Aurélien Mosson ${ }^{\S}$, Morad Kassem ${ }^{\text {Il }}$ \\ Airbus Operations S.A.S, 316 Route de Bayonne, 31300 Toulouse, France. \\ Benoit Van Den Nieuwenhof ${ }^{\|}$ \\ Free Field Technologies, Rue Emile Francqui 9, 1435 Mont-Saint-Guibert, Belgique.
}

This paper is devoted to the uncertainty quantification for 3D acoustic performance model of nacelle liners (acoustic treatments). Uncertainties are taken into account in order to increase the robustness of the predictions. A full computational acoustic propagation model based on the convected Helmholtz equation in presence of a non-homogeneous flow velocity field computed by solving the Linearized Euler Equations (LEE) is used. A reduced-order computational model is deduced in order to implement the probabilistic model of uncertainties. The model uncertainties induced by modeling errors have been taken into account for the acoustic propagation model and the liner model, using the nonparametric probabilistic approach. In addition, the uncertainties on the acoustic excitation induced by the fan have been introduced using the parametric probabilistic approach. The developed methodology is applied to a 3D nacelle intake and allows for computing the confidence regions of the random far-field radiated pressure in terms of random SPL (Sound Pressure Level), which are compared to experiments for several flight conditions and frequencies.

\section{Nomenclature}

\footnotetext{
$\mathbf{r}(x, y, z), t, \omega$

$\tilde{X}, X_{0}, X$

$M_{\infty}, M$

$c_{\alpha}, \varphi_{\alpha}(x, y), k_{z_{\alpha}}, k_{x y_{\alpha}}$

$=$ Uniform and local Mach number

$\mathcal{S}_{\text {duct }}$

$\tilde{\rho}, \rho_{0}, \rho_{\infty}, \rho$

$=$ Cross section of the duct domain $\Omega_{\text {duct }}$

$\tilde{\mathbf{v}}, \mathbf{v}_{0}, \mathbf{v}_{\infty}, \mathbf{v}$

$\tilde{p}, p_{0}, p$

$\Omega_{\mathrm{i}}, \Omega_{\mathrm{o}}, \Omega_{\text {duct }}$

$\partial \Omega_{\mathrm{i}}, \partial \Omega_{\mathrm{o}}, \partial \Omega_{\mathrm{duct}}$

$\Gamma_{\text {io }}, \Gamma_{\mathrm{h}}, \Gamma, \Gamma_{\mathrm{f}}, \Gamma_{\text {ho }}, \Gamma_{\text {duct }}$

$\mathbf{n}_{\text {io }}, \mathbf{n}_{\mathrm{h}}, \mathbf{n}, \mathbf{n}_{\mathrm{f}}, \mathbf{n}_{\text {ho }}, \mathbf{n}_{\text {duct }}$

$=$ Total, steady-flow, uniform, and fluctuating fluid velocity

$=$ Total, steady-flow, and fluctuating pressure fields

$=$ Inner, outer, and duct domains of the intake acoustic problem

$=$ Inner, outer, duct domain boundaries

$=$ Outward unit normal of surfaces $\Gamma_{\mathrm{io}}, \Gamma_{\mathrm{h}}, \Gamma, \Gamma_{\mathrm{f}}, \Gamma_{\mathrm{ho}}, \Gamma_{\text {duct }}$,

$\psi_{\mathrm{i}}, \psi_{\mathrm{o}}, \varphi$

*PhD Student, Université Gustave Eiffel, MSME UMR 8208 CNRS, vincent.dangla@airbus.com

†Professor, Université Gustave Eiffel, MSME UMR 8208 CNRS, christian.soize@univ-eiffel .fr

${ }^{\ddagger}$ Research Engineer, Airbus Operations S.A.S, morad.kassem@airbus.com

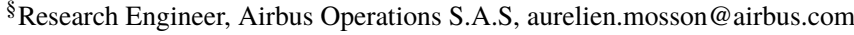

"I Research Engineer, Airbus Operations S.A.S, morad.kassem@airbus.com

" Senior Acoustic Consultant, Free Field Technologies, Benoit.VanDenNieuwenhof@fft.be
}

$=$ Space three-dimensional vector, time scalar variable, and angular frequency

$=$ Total, static, and fluctuating thermodynamic variable, function of space and time

$=$ Amplitude, eigenfunction, axial, and transversal wave numbers of a duct mode $\alpha$

$=$ Total, steady-flow, uniform, and fluctuating fluid mass density

$=$ Interface between inner and outer domains, hardwall, liner, fan, outer hardwall, and duct surfaces

$=$ Acoustic velocity potential in the inner, outer, and duct domains 


$\begin{array}{ll}Z(\mathbf{r}, \omega), Z_{R}(\mathbf{r}, \omega), Z_{I}(\mathbf{r}, \omega) & =\text { Liner local impedance, resistance and reactance } \\ \mathbf{v}_{\Gamma}(\mathbf{r}, \omega) & =\text { Wall acoustic velocity for the liner (Myers boundary condition) } \\ \Delta_{x y} & =\text { Laplacian in the transversal plane }(x, y) \\ S_{f}(\mathbf{r}, \omega) & =\text { Acoustic source term induced by then fan rotation } \\ {\left[Z_{\mathrm{i}}\right]} & =\text { Coupling matrix between inner and outer domain finite element discretization } \\ {[H(\omega)]} & =\text { Mass and stiffness matrix of the pellicular domain } \\ {\left[\mathcal{M}_{L}\right],\left[\mathcal{K}_{L}\right]} & =\text { Aeroacoustic, liner matrices, and excitation vector condensed on liner dofs } \\ {\left[\mathcal{A}_{\ell}\right],\left[\mathcal{Z}_{\ell}\right], \mathcal{F}_{\ell}} & =\text { Generalized coordinates of the Reduced-Order Model } \\ \mathbf{q} & =\text { Generalized aeroacoustic, liner matrices, and excitation vector } \\ {\left[\mathbb{A}_{1}\right],[\mathbb{Z}], \mathbb{F}} & =\text { Imaginary and real parts of the generalized Aeroacoustic matrix } \\ {\left[\mathbb{A}_{R}\right],\left[\mathbb{A}_{I}\right]} & =\text { Random real and imaginary part of the generalized Aeroacoustic matrix } \\ {\left[\mathbf{A}_{R}\right],\left[\mathbf{A}_{I}\right]} & =\text { Imaginary, real, skew-symmetric imaginary, symmetric imaginary, } \\ {\left[\mathbb{Z}_{R}\right],\left[\mathbb{Z}_{I}\right],\left[\mathbb{Z}_{I}^{\text {sS }}\right],\left[\mathbb{Z}_{I}^{\mathrm{S}}\right],\left[\mathbb{Z}^{\mathrm{NS}}\right]} & =\text { nonsymmetric parts of the generalized Liner matrix } \\ & =\text { Random real, imaginary, skew-symmetric imaginary, symmetric imaginary, } \\ {\left[\mathbf{Z}_{R}\right],\left[\mathbf{Z}_{I}\right],\left[\mathbf{Z}_{I}^{\text {SS }}\right],\left[\mathbf{Z}_{I}^{\mathrm{S}}\right],\left[\mathbf{Z}^{\mathrm{NS}}\right]} & =\text { nonsymmetric parts of the generalized Liner matrix } \\ & =\text { Vector of the quantities of interest } \\ \text { QoI } & =\text { Number of dofs in the inner, outer domains, fan surface, and number of cut-on modes } \\ N_{\mathrm{i}}, N_{\mathrm{o}}, N_{\mathrm{f}}, N_{\mathrm{d}} & =\text { Number of liner dofs, number of pellicular modes } \\ N_{\ell}, n_{\ell} & =\text { Transfer matrix of zeros and ones selecting non zeros dofs related to fan excitation } \\ {\left[\varepsilon_{\mathrm{if}}\right]} & =\text { Vector of incident duct modes amplitudes } \\ \mathbf{c}^{+} & \\ \text {Subscripts } & =\text { Linearized Euler Equations } \\ \text { LEE } & =\text { Degrees of freedom } \\ \text { Dofs } & =\text { Quantities of Interest } \\ \text { QoIs } & =\text { Sound Pressure Level } \\ \text { SPL } & \end{array}$

\section{Introduction}

$\mathbf{T}^{\mathrm{N}}$ modern turbofan engines, fan noise is one of the main sources due to increased bypass ratio of the engines. Acoustic liners (acoustic treatments) are effective in mitigating noise generated by the fan. In order to be efficient, namely to absorb the fan noise, liners have to be studied in their operating conditions. A scheme of the generic mechanical system involving the fan, the liner, and the nacelle intake is shown in Figure1. The acoustic source is the fan and we are interested in predicting the far-field radiated pressure as a function of the liner acoustic properties. As the liner design is frozen in early phases of aircraft development, the maturity of aircraft data is relatively low, which implies a non-negligible variability on liner design parameters. In order to account for this variability and increase the robustness of the predictions performed using a 3D computational model of the convected acoustic propagation, a robust design of the liner is carried out, in which modeling uncertainties in the propagation and liner models and parametric uncertainties for the fan acoustic excitation are accounted for. The finality of such a tool would be to associate liner acoustic performance and robustness by giving statistical information about the pressure response of a given design. This work is devoted to the analysis of the convected acoustic propagation in the framework of lined nacelle intakes, in presence of uncertainties. The aeroacoustic model is based on the Möhring analogy [1], in which the acoustic sources are not accounted for since flow-induced noise is negligible with respect to the fan-generated noise. In order to ease the writing, the word aeroacoustic refers, in this paper, to the convected acoustic propagation model. As the walls are fixed (no wall displacement), the formulation for the acoustic propagation in the moving fluid is performed using the linear convected Helmholtz equation for which the velocity field of the steady flow is computed by solving the Euler potential equation. The liner performance is assessed using computational models that include a modeling of uncertainties for the aeroacoustic model, for the liner model, and for the acoustic excitation induced by the fan. These uncertainties are modeled in the framework of the probability theory using the nonparametric probabilistic approach involving random matrix theory [2] and the parametric probabilistic method. Some works devoted to uncertainty quantification for the liner impedance in presence of a flow have also been carried out in the last decade (see for instance [3-7]).

The paper is organized as follows. In Section [I] we present the nominal computational model. This computational model includes the convected acoustic propagation model and the liner model. This nominal computational model corresponds 


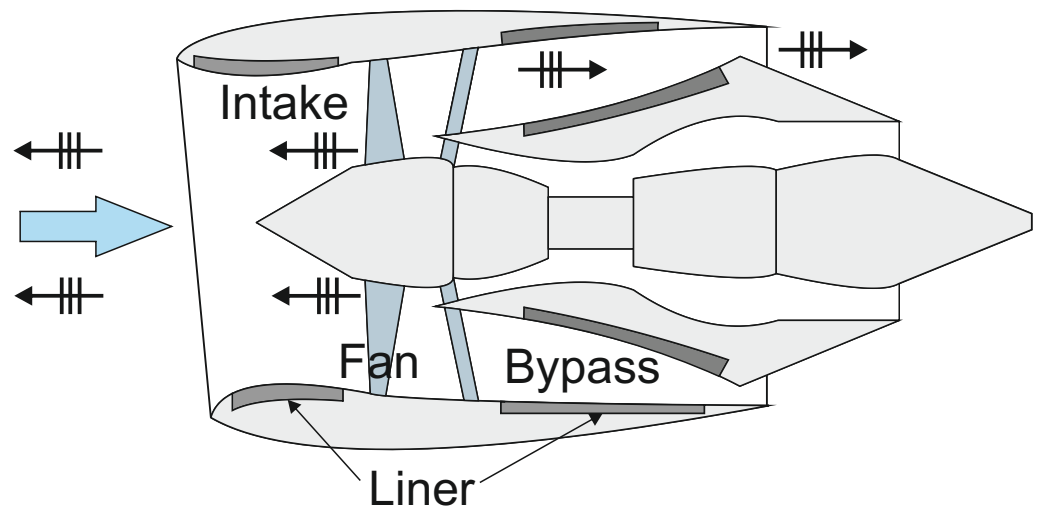

Fig. 1 Scheme of a generic 3D nacelle

to the finite/infinite element discretization of the weak formulation of the boundary value problem. Section [III deals with the construction of a computational reduced-order model that is required for reducing the numerical cost and above all for allowing the nonparametric probabilistic approach of uncertainties to be implemented. This reduced-order model is constructed using a modal basis that is associated with the acoustic radiation in the acoustic layer (pellicular domain) over lined surfaces. In Section IV, we present the probabilistic model for uncertainty quantification. It consists in constructing a probabilistic model for the aeroacoustic model, for the liner model, and for the acoustic excitation induced by the fan. Section $\nabla$ is devoted to the presentation of the configuration and of the computational model of a generic industrial 3D nacelle. SectionVIII deals with a sensitivity analysis of modal SPL with respect to the levels of uncertainties. Finally, in Section IX, we present the robust predictions of the SPL with an experimental validation.

\section{Construction of the nominal computational model}

\section{A. Summarizing the methodology for constructing the nominal deterministic computational model}

First, we will present the boundary value problem associated with 3D inner, outer, and duct domains of the intake acoustic radiation problem. The linear convected Helmhlotz equation [1, 8-11] is used in the inner domain (near-field) and in the outer domain (far-field), involving the velocity and mass density fields of the flow computed using LEE. The boundary condition on the liner is the Myers condition [12-14] involving the local admittance of the liner model [15] depending on the spatial point and on the frequency. At infinity, the outward Sommerfeld radiation condition is written. For the fan excitation (see for instance [16] and [17]), the acoustic velocity field is represented on the family of acoustic duct modes and allows for ensuring the coupling between the semi-infinite duct and the inner domain on the fan boundary, and for representing the acoustic excitation produced by the fan. It should be noted that the wall of the duct is rigid and its cross-section is assumed to be constant. Inside the duct, the flow is assumed to be uniform. Consequently, the duct modes are explicitly known.

Then, the finite/infinite element discretization of the weak formulation of the boundary value problem is performed, which allows for obtaining the computational model in the frequency domain. The unknowns of this computational model are the discretized acoustic velocity potential fields in the inner domain and in the outer domain, and the vector of the coefficients describing the unknown reflected sound field. In this computational model, the acoustic source term depends on the vector of the known coefficients of the incident sound field describing the fan acoustic excitation.

Finally, for each frequency, we introduce the computational model that is a very large linear complex matrix system and that induces a large computational cost when exploring the frequency band of analysis. In addition, the nonparametric probabilistic approach of uncertainties [2, 18] has to be implemented in the computational model. For these two reasons, a computational reduced-order model depending on the frequency is constructed. For this purpose, two ingredients are introduced [19, 20]. The first one is a frequency by frequency static condensation of the aeroacoustic degrees of 
freedom on the liner dofs. Since the number of liner dofs is generally large, the reduced-order model is constructed by introducing a pellicular basis constituted of a set of acoustic modes in a pellicular domain that is related to liner dofs. All this methodology has been implemented in a commercial software [19, 21, 22], which will be used for the numerical simulations presented in Section VIII

\section{B. Definition of the geometry of the acoustic problem}

For liner performance assessment, an aeroacoustic modeling of the acoustic radiation problem of a nacelle intake is used. The boundary value problem associated with this model (see Fig. 1 and Fig. 2) is formulated using three domains. In Fig. 2. a first domain $\Omega_{\mathrm{i}}$ corresponds to the nacelle near-field, which is discretized by finite elements. A second domain $\Omega_{\mathrm{o}}$ corresponds to the far-field, which is discretized by infinite elements. A third domain $\Omega_{\text {duct }}$ allows for analytically calculating the acoustic excitation imposed by the fan, for which the model is based on analytical duct modes ([16]). In order to numerically solve the intake acoustic radiation problem, the associated boundary value problem must be written involving a propagation equation and boundary conditions. For a given flight condition, the flow computation assumes that the fluid is inviscid (viscous and thermal effects are neglected) and is performed by solving potential Euler equations on an appropriate CFD mesh. From these CFD computations, the steady-flow velocity field $\mathbf{v}_{0}$ and the steady-flow mass density field $\rho_{0}$ are deduced. The convected acoustic wave equation comes from the derivation of the Navier-Stokes equations ([16], [8]), for which it is assumed an irrotational locally isentropic flow. The linearized convected acoustic equation is written in the frequency domain (assuming that fluctuating thermodynamic quantities are small compared to their mean-flow counterpart).

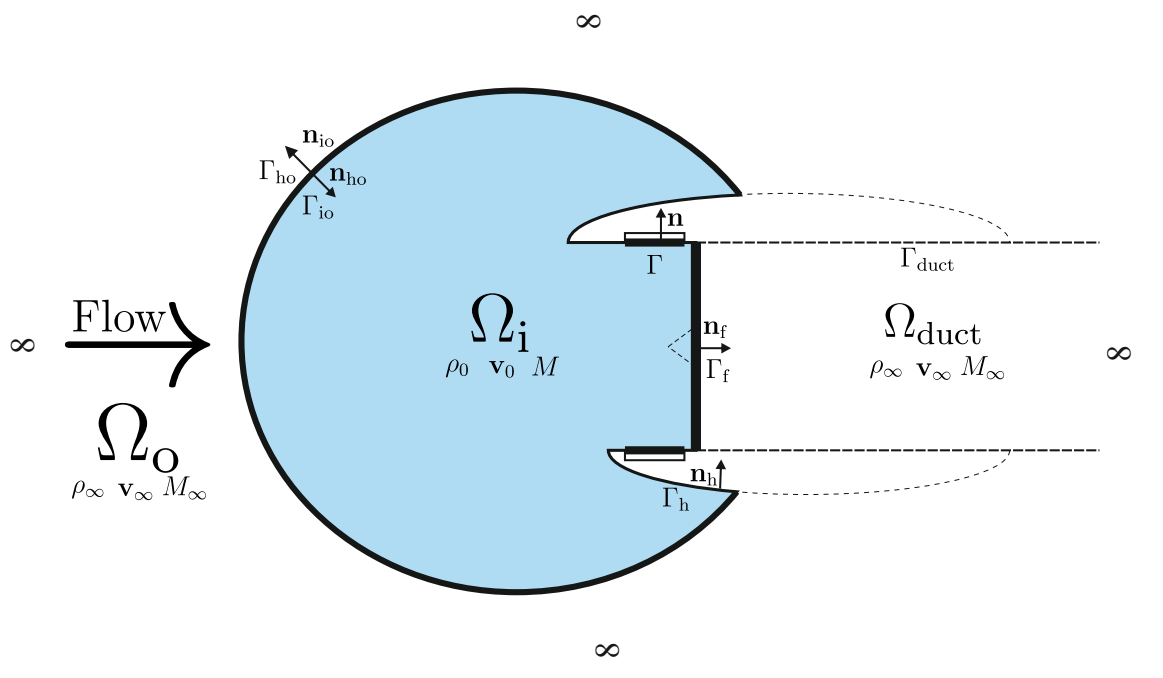

Fig. 2 2D section of the half-3D geometry for the boundary value problem of the intake radiation problem in presence of a flow, corresponding to the nacelle scheme in Fig 1 .

Figure 2 is a $2 \mathrm{D}$ section of the half-3D geometry of the inlet acoustic problem, in which $\mathbb{R}^{3}$ is the whole domain, $\Omega_{\mathrm{i}}$ is the inner bounded open domain of $\mathbb{R}^{3}$ of boundary $\partial \Omega_{\mathrm{i}}=\Gamma_{\mathrm{io}} \cup \Gamma_{\mathrm{h}} \cup \Gamma \cup \Gamma_{\mathrm{f}}$, and $\Omega_{\mathrm{o}}=\mathbb{R}^{3} \backslash \bar{\Omega}_{\mathrm{i}}$ is the outer unbounded open domain of $\mathbb{R}^{3}$, whose boundary $\partial \Omega_{\mathrm{o}}$. The generic point in $\mathbb{R}^{3}$ is denoted as $\mathbf{r}=(x, y, z)$, for which the direction of coordinate $z$ is the one of the rotation axis of the fan.

Definition of the geometry and boundary conditions for inner domain $\Omega_{\mathrm{i}}$. The unit normal to boundary $\partial \Omega_{\mathrm{i}}$ outward of domain $\Omega_{\mathrm{i}}$ is written as, $\mathbf{n}_{\mathrm{io}}$ for $\Gamma_{\mathrm{io}}, \mathbf{n}_{\mathrm{h}}$ for $\Gamma_{\mathrm{h}}, \mathbf{n}$ for $\Gamma$, and $\mathbf{n}_{\mathrm{f}}$ for $\Gamma_{\mathrm{f}}$. Part $\Gamma$ of the boundary $\partial \Omega_{\mathrm{o}}=\partial \Omega_{\mathrm{i}}$ is the coupling interface between the acoustic part $\Omega_{\mathrm{i}}$ and the liner, while $\Gamma_{\mathrm{f}}$ is the part on which the fan excitation is applied. The inner problem implies the use of the following boundary conditions:

- $\Gamma_{\text {io }}$ (inner outer) represents a nonphysical interface between inner and outer domains, on which the continuity condition is written for the acoustic velocity potential and for its gradient.

- $\Gamma_{\mathrm{h}}$ (hardwall) corresponds to rigid or "hardwall" part characterized by a zero normal acoustic velocity.

- $\Gamma$ (liner) corresponds to an acoustic treatment characterized by its impedance.

- $\Gamma_{f}$ (fan) corresponds to the part of the boundary on which act acoustic sources induced by the fan. 
Definition of the geometry and boundary conditions for outer domain $\Omega_{0}$. The boundary $\partial \Omega_{0}$ of $\Omega_{0}$ is written as $\Gamma_{\text {io }} \cup \Gamma_{\text {ho }}$ in which $\Gamma_{\text {ho }}=\Gamma_{\mathrm{h}} \cup \Gamma \cup \Gamma_{\mathrm{f}}$ (this means that $\partial \Omega_{\mathrm{o}}=\partial \Omega_{\mathrm{i}}$ ). The unit normal to boundary $\partial \Omega_{\mathrm{o}}$ outward of domain $\Omega_{\mathrm{o}}$ is written as, $\mathbf{n}_{\mathrm{oi}}=-\mathbf{n}_{\mathrm{io}}$ for the part $\Gamma_{\mathrm{io}}$ and $\mathbf{n}_{\mathrm{ho}}$ for the part $\Gamma_{\mathrm{ho}}$. For the acoustic wave propagation in the outer domain, $\Gamma_{\text {ho }}$ corresponds to a rigid wall on which the normal acoustic velocity will be equal to zero.

The outer problem implies the use of the following boundary conditions:

- $\Gamma_{\text {io }}$ (inner outer) is a nonphysical coupling interface between inner and outer domains, on which the continuity is written for the acoustic velocity potential and for its gradient (note that infinite boundary elements will be used on $\Gamma_{\text {io }}$ for accounting for the effects of $\Omega_{\mathrm{o}}$ on $\Omega_{\mathrm{i}}$ through the coupling interface $\Gamma_{\text {io }}$ ).

- $\Gamma_{\text {ho }}$ (hardwall outer) corresponds to a rigid wall characterized by a zero normal acoustic velocity.

Definition of the geometry and boundary conditions for the semi-infinite duct $\Omega_{\text {duct }}$. A semi-infinite cylindrical duct $\Omega_{\text {duct }}$ is connected to the inner domain $\Omega_{\mathrm{i}}$ for which the coupling interface is the fan plane $\Gamma_{f}$. The boundary $\partial \Omega_{\text {duct }}$ of semi-infinite domain $\Omega_{\text {duct }}$ is written as $\partial \Omega_{\text {duct }}=\Gamma_{f} \cup \Gamma_{\text {duct }}$ in which $\Gamma_{\text {duct }}$ is the cylindrical-surface part of the boundary of $\Omega_{\text {duct }}$. It should be noted that this domain is only introduced for generating the acoustic excitation induced by the fan and consequently, has to be viewed as a "fictional domain" with respect to the formulation of the boundary value problem related to $\overline{\Omega_{\mathrm{i}} \cup \Omega_{\mathrm{o}}}$. This is the reason why $\Omega_{\mathrm{o}}=\mathbb{R}^{3} \backslash \bar{\Omega}_{\mathrm{i}}$. In this semi-infinite duct, the sound field is the sum of a given incident sound field and an unknown reflected sound field, which will be represented on the duct modes (see Section II.C.2. The wall of the boundary of $\Omega_{\text {duct }}$ is rigid on which a zero normal acoustic velocity is applied.

\section{Boundary value problem associated with innner, outer, and duct domains of the intake acoustic radiation problem}

In this subsection we only present the most important concepts and equations associated with the boundary value problem, for which the definitions and the notations introduced in Section II.B are used.

\section{Boundary value problem in the inner and outer domains $\Omega_{i}$ and $\Omega_{o}$ (near-field and far-field)}

Linear convected Helmholtz equation in $\Omega_{\mathbf{i}}$ and $\Omega_{\mathbf{o}}$. The linear frequency formulation of the convected wave equation is written in $\Omega_{\mathrm{i}}([1,8,-11])$, under the following hypotheses: no aerodynamic acoustic source terms, no heat production due to viscous dissipation, no heat transfers in the flow, no gravity forces, and the flow is locally isentropic. The convected wave equation is then written as,

$$
-\frac{\omega^{2} \rho_{0}}{c^{2}} \psi_{\mathrm{i}}+\frac{j \omega \rho_{0}}{c^{2}} \mathbf{v}_{0} \cdot \boldsymbol{\nabla} \psi_{\mathrm{i}}+\boldsymbol{\nabla} \cdot\left(\frac{j \omega \rho_{0} \mathbf{v}_{0}}{c^{2}} \psi_{\mathrm{i}}+\frac{\rho_{0} \mathbf{v}_{0}}{c^{2}} \mathbf{v}_{0} \cdot \boldsymbol{\nabla} \psi_{\mathrm{i}}-\rho_{0} \boldsymbol{\nabla} \psi_{\mathrm{i}}\right)=0,
$$

in which the field $\psi_{\mathrm{i}}$ is the acoustic velocity potential in inner domain $\Omega_{\mathrm{i}}$, linked to the acoustic pressure and velocity fields by the equation,

$$
p=-j \omega \rho_{0} \psi_{\mathrm{i}}-\rho_{0} \mathbf{v}_{0} \cdot \boldsymbol{\nabla} \psi_{\mathrm{i}} \quad, \quad \mathbf{v}=\boldsymbol{\nabla} \psi_{\mathrm{i}}
$$

In the above equations, $c$ is the speed of sound at equilibrium, $\omega$ is the angular frequency $(\mathrm{rad} / \mathrm{s}), j=\sqrt{-1}$ is the pure imaginary complex number, $\boldsymbol{\nabla}$ is the gradient operator with respect to space variable $\mathbf{r}=(x, y, z)$, and where the symbol "dot" represents the Euclidian inner product in $\mathbb{R}^{3}$.

In the outer domain, the same convected Helmholtz equation is used, in which mean flow quantities are replaced by $\rho_{\infty}$ and $\mathbf{v}_{\infty}$, which are the constant mass density and velocity fields of the uniform inflow in the z-direction.

Myers boundary condition related to the liner surface (inner domain $\Omega_{\mathbf{i}}$ ). The boundary condition on $\Gamma$ (liner) is written $([12],[13])$ as ,

$$
\frac{\partial \psi_{i}}{\partial \mathbf{n}}=\left(1-\frac{1}{j \omega} \mathbf{v}_{0} \cdot \boldsymbol{\nabla}+\frac{1}{j \omega} \mathbf{n} \cdot(\mathbf{n} \cdot \boldsymbol{\nabla}) \mathbf{v}_{0}\right)\left(\frac{1}{Z(\mathbf{r}, \omega)}\left(j \omega \rho_{0} \psi_{\mathrm{i}}+\rho_{0} \mathbf{v}_{0} \cdot \boldsymbol{\nabla} \psi_{\mathrm{i}}\right)\right),
$$

in which $\frac{1}{Z(\mathbf{r}, \omega)}$ denotes the local admittance at point $\mathbf{r}$ and at frequency $\omega$ of the liner, and where $\mathbf{n}$ is the unit normal to $\Gamma$ external to $\Omega_{\mathrm{i}}$. 


\section{Boundary value problem in the semi-infinite duct $\Omega_{\text {duct }}$ for the inlet case and duct modes}

For the fan excitation (see for instance [16] and [17]), the acoustic velocity potential field $\varphi$ in $\Omega_{\text {duct }}$ will be represented on the family of acoustic duct modes and will allow (i) for ensuring the coupling between the semi-infinite duct $\Omega_{\text {duct }}$ and the inner domain $\Omega_{\mathrm{i}}$ on boundary $\Gamma_{f}$ (see Fig. 2), and (ii) for representing the acoustic excitation produced by the fan. In this work, it is assumed that $\Omega_{\text {duct }}$ is a straight cylinder with $z$-axis, for which its cross-section is constant and consequently, independent of $z$. The steady flow in $\Omega_{\text {duct }}$ is assumed to be subsonic and uniform along the $z$-direction.

Linear convected Helmholtz equation in $\Omega_{\text {duct }}$. We consider the acoustic propagation in $z$-direction. The acoustic velocity potential $\varphi(x, y, z)$ verifies the following linear convected Helmholtz equation,

$$
\Delta_{x y} \varphi+\left(1-M^{2}\right) \frac{\partial^{2} \varphi}{\partial z^{2}}-2 j k M \frac{\partial \varphi}{\partial z}+k^{2} \varphi=0 \quad \text { in } \quad \Omega_{\text {duct }},
$$

where $\Delta_{x y}$ is the Laplacian in the transversal plane $(x, y), M=\left\|\mathbf{v}_{\infty}\right\| / c$ is the Mach number associated to the steady flow, $k=\omega / c$ is the wave number.

The boundary condition on $\Gamma_{\text {duct }}$ consists in writing that the normal derivative of the acoustic velocity potential is zero. In addition, the continuity on interface $\Gamma_{f}$ of acoustic velocity potential $\varphi$ with acoustic velocity potential $\psi_{\mathrm{i}}$ is written as $\varphi=\psi_{\mathrm{i}}$ on $\Gamma_{f}$.

Modal representation for the acoustic excitation by the fan and the reflected sound field. Using Rienstra's work [23] on constant cross-section ducts (independent of $z$ ), the acoustic velocity potential field $\varphi$ introduced in Eq. (4) is rewritten by keeping only a finite number $N_{d}$ of duct modes and by showing the contribution of the given incident sound field (represented by symbol +) that represents the acoustic excitation induced by the fan and the contribution of the unknown associated reflected sound field (represented by symbol -). From Eq. (4), function $\varphi(x, y, z)$ can be written as,

$$
\varphi(x, y, z)=\sum_{\alpha=1}^{N_{d}} \varphi_{\alpha}(x, y)\left(c_{\alpha}^{+} e^{j k_{z_{\alpha}}^{+} z}+c_{\alpha}^{-} e^{j k_{z_{\alpha}}^{-} z}\right),
$$

in which $k_{z_{\alpha}}^{+}$is the axial wavenumber of the wave propagating in the upstream direction and $k_{z_{\alpha}}^{-}$is the axial wavenumber of the wave propagating in the downstream direction.

It should be noted that the acoustic excitation by the fan, which is associated with the incident sound field, will be defined by giving the complex vector $\mathbf{c}^{+}=\left(c_{1}^{+}, \ldots, c_{N_{d}}^{+}\right)$, while the unknown reflected sound field depends on the vector $\mathbf{c}^{-}=\left(c_{1}^{-}, \ldots, c_{N_{d}}^{-}\right)$and should be included with all the unknown fields of the acoustic problem.

Definition of the modal intensity $I_{\alpha}^{+} \quad$ In the present paragraph, the frequency $\omega$ is fixed and is removed for simplifying the writing. The acoustic pressure $p$ is related to the velocity potential $\varphi$ by Eq. (2) and the continuity condition $\psi_{\mathrm{i}}=\varphi$ on $\Gamma_{\mathrm{f}}$. The velocity potential $\varphi$, can be rewritten as $\varphi=\varphi^{+}+\varphi^{-}$, where

$$
\varphi^{+}(x, y, z)=\sum_{\alpha} c_{\alpha}^{+} \varphi_{\alpha}(x, y) e^{j k_{z \alpha} z} .
$$

We then define $p_{\alpha}^{+}$,

$$
p_{\alpha}^{+}=-j \omega \rho_{0} \varphi_{\alpha}^{+}-\rho_{0} \mathbf{v}_{0} \cdot \nabla \varphi_{\alpha}^{+} .
$$

in which $\varphi_{\alpha}^{+}$is the contribution of mode $\alpha$ in $\varphi^{+}$. The modal intensity $I_{\alpha}^{+}$, related to pressure $p_{\alpha}^{+}$, is defined by

$$
I_{\alpha}^{+}=10 \log _{10}\left(\frac{\left|p_{\alpha}^{+}\right|^{2}}{p_{\text {ref }}^{2}}\right),
$$

in which $p_{\text {ref }}=2 \times 10^{-5}$ is the reference pressure. 
Modeling of the nominal modal intensity $\underline{I}_{\alpha}^{+} \quad$ Simulated data are computed following hypotheses on the modal content that concern broadband and emergence levels, and energy repartition between the different modes. For that purpose, a custom modal content is introduced, based on several sate-of-the-art modal contents. A broadband level is set for all azimuthal and radial orders. One emergence level corresponding to the engine order is set, as well as two other emergence levels for which the azimuthal order can be set. Then, a number of azimuthal orders from each side of each emergence (corresponding to the impact of emergences over neighboring modes), for which the acoustic energy decays as a function of the azimuthal order. For all emergences, the energy is only carried by the first radial order. Algebraically, the modal content can be described using the following parameters. An example can be found on the figure below.

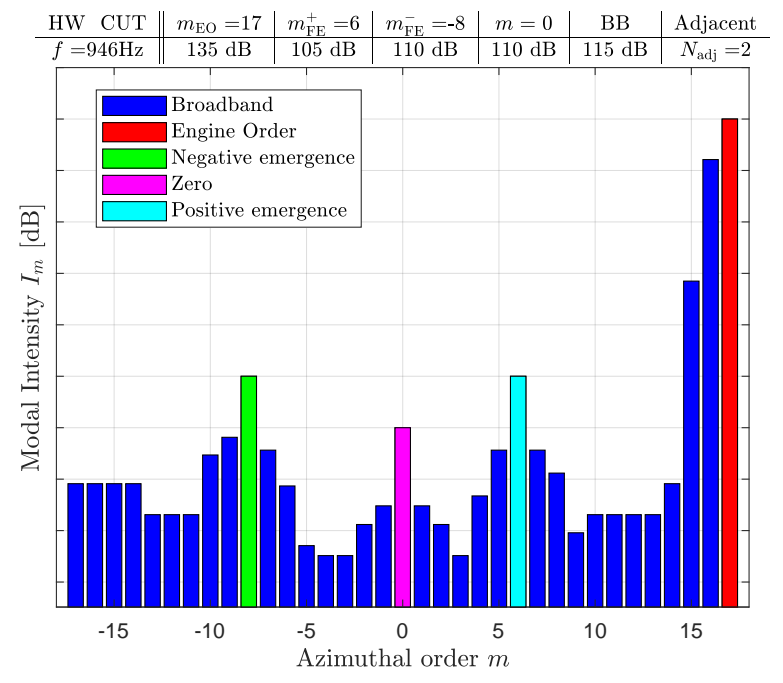

- $I_{m n}$ is the modal intensity of azimuthal order $m$ and radial order $n$,

- $I_{\mathrm{EO}}$ is the modal intensity of the engine order of azimuthal order $m=m_{\mathrm{EO}}$ (red bar),

- $I_{\mathrm{FE}^{-}}$is the modal intensity of the negative flexible emergence of azimuthal order $m=m_{\mathrm{FE}^{-}}$(green bar),

- $I_{\mathrm{FE}^{+}}$is the modal intensity of the quarter engine order of azimuthal order $m=m_{\mathrm{FE}^{+}}$(cyan bar),

- $I_{\mathrm{BB}}$ is the modal intensity of the broadband, $N_{\text {adj }}$ is the number of adjacent modes on which the engine order acoustic energy is reverberated (blue bar),

- $N_{\text {azi }}$ is the number of azimuthal orders, and $N_{\text {rad }}$ is the number of radial orders per azimuthal order.

\section{Finite/Infinite Element discretization of the weak formulation}

The weak formulation of the boundary value problem defined by Eqs (17) to (4) with $\varphi$ given by (5) is discretized by the Finite/Infinite Element Method (FEM/IEM). For all $\omega$ fixed in $\mathbb{R}$, let $\psi_{\mathrm{i}}(\omega)$ be the complex vector in $\mathbb{C}^{N_{\mathrm{i}}}$ corresponding to the finite element discretization of field $\psi_{\mathrm{i}}(\mathbf{r}, \omega)$ in which $N_{\mathrm{i}}$ is the number of degrees of freedom (dofs). Let $\psi_{\mathrm{o}}(\omega)$ be the complex vector in $\mathbb{C}^{N_{\mathrm{o}}}$ corresponding to the infinite element discretization of field $\psi_{\mathrm{o}}(\mathbf{r}, \omega)$ in which $N_{\mathrm{o}}$ is the number of dofs in the outer domain. We then obtain the computational model in terms of $\boldsymbol{\psi}_{\mathrm{i}}(\omega), \boldsymbol{\psi}_{\mathrm{o}}(\omega)$, and $\mathbf{c}^{-}(\omega)$, which is written as,

$$
\left[\begin{array}{ccc}
{\left[A_{\mathrm{i}}(\omega)\right]+\left[Z_{\mathrm{i}}(\omega)\right]} & {[H(\omega)]} & -\left[\mathcal{E}_{\mathrm{if}}\right]\left[F^{-}(\omega)\right] \\
-[H(\omega)]^{T} & {\left[A_{\mathrm{o}}(\omega)\right]} & {[0]} \\
{[E]} & {[0]} & -\left[R^{-}(\omega)\right]
\end{array}\right]\left[\begin{array}{c}
\boldsymbol{\psi}_{\mathrm{i}}(\omega) \\
\boldsymbol{\psi}_{\mathrm{o}}(\omega) \\
\mathbf{c}^{-}(\omega)
\end{array}\right]=\left[\begin{array}{c}
{\left[\mathcal{E}_{\mathrm{if}}\right]\left[F^{+}(\omega)\right]} \\
{[0]} \\
{\left[R^{+}(\omega)\right]}
\end{array}\right] \mathbf{c}^{+},
$$

in which,

- the $\left(N_{\mathrm{i}} \times N_{\mathrm{i}}\right)$ complex matrix $\left[A_{\mathrm{i}}(\omega)\right]$, the $\left(N_{\mathrm{o}} \times N_{\mathrm{o}}\right)$ complex matrix $\left[A_{\mathrm{o}}(\omega)\right]$, and the $\left(N_{\mathrm{i}} \times N_{\mathrm{o}}\right)$ complex matrix $[H(\omega)]$ correspond to the block decomposition of the aeroacoustic impedance matrix.

- The diagonal $\left(N_{\mathrm{d}} \times N_{\mathrm{d}}\right)$ complex matrices $\left[R^{+}(\omega)\right]$ and $\left[R^{-}(\omega)\right]$ correspond to the incident and to the reflected modal participations in the duct domain $\Omega_{\text {duct }}$.

- The $\left(N_{\mathrm{d}} \times N_{\mathrm{i}}\right)$ constant real matrix $[E]$ corresponds to the finite element discretization of the inner product in the space of all the square integrable functions on boundary $\Gamma_{\mathrm{f}}$.

- The $\left(N_{\mathrm{i}} \times N_{\mathrm{f}}\right)$ real matrix [ $\left.\mathcal{E}_{\mathrm{if}}\right]$ constituted of ones and zeros, allows for selecting the dofs related to boundary $\Gamma_{\mathrm{f}}$.

- The $\left(N_{\mathrm{f}} \times N_{\mathrm{d}}\right)$ complex matrices $\left[F^{+}(\omega)\right]$ and $\left[F^{-}(\omega)\right]$ represent the discretization of the incident and the reflected modal participations in the inner domain $\Omega_{\mathrm{i}}$.

\section{Computational reduced-order model}

In Section [V] we will present the probabilistic modeling of uncertainties in the computational model defined by Eq. 9]. These uncertainties will be taken into account for both the parametric uncertainties and the model uncertainties induced by the modeling errors for the aeroacoustic and for the liner. Consequently, we need to reorganize the algebraic structure 
of the complex matrix equation defined by Eq. (9) in order to exhibit the unknowns related to the liner boundary, and thus create an interface problem between the liner and the rest of the model. Moreover, since the nonparametric approach of uncertainties is used, the system size needs to be drastically reduced. This is why we use the static condensation coupled with a reduced-order model.

\section{A. Frequency by frequency static condensation with respect to the liner dofs and expression of the fan acoustic excitation}

the complex vector $\psi_{\ell}(\omega)$ belonging to $\mathbb{C}^{N_{\ell}}$ of the $N_{\ell}$ dofs of the liner and the complex vector $\psi_{a}$ belonging to $\mathbb{C}^{N_{a}}$ of the $N_{a}$ other dofs of $\left(\boldsymbol{\psi}_{\mathrm{i}}(\omega), \boldsymbol{\psi}_{\mathrm{o}}(\omega), \mathbf{c}^{-}(\omega)\right)$. This means that $N_{a}=N_{\mathrm{i}}+N_{\mathrm{o}}+N_{d}-N_{\ell}$.

The computational model defined by Eq. (9) can be rewritten in a block form as,

$$
\left[\begin{array}{ll}
{\left[A_{\ell \ell}(\omega)\right]} & {\left[A_{\ell a}(\omega)\right]} \\
{\left[A_{a \ell}(\omega)\right]} & {\left[A_{a a}(\omega)\right]}
\end{array}\right]\left[\begin{array}{l}
\psi_{\ell}(\omega) \\
\psi_{a}(\omega)
\end{array}\right]+\left[\begin{array}{cc}
{\left[Z_{\ell}(\omega)\right]} & 0 \\
0 & 0
\end{array}\right]\left[\begin{array}{l}
\psi_{\ell}(\omega) \\
\psi_{a}(\omega)
\end{array}\right]=\left[\begin{array}{c}
\mathbf{0} \\
\mathbf{f}_{a}(\omega)
\end{array}\right]
$$

An $\omega$ by $\omega$ static condensation using the Schur complement method (see for instance [20]) is performed by eliminating the vector $\psi_{a}(\omega)$, which yields,

$$
\left(\left[\mathcal{A}_{\ell}(\omega)\right]+\left[Z_{\ell}(\omega)\right]\right) \psi_{\ell}(\omega)=\left[\mathcal{B}_{\ell}(\omega)\right] \mathbf{c}^{+} .
$$

in which $\left[\mathcal{A}_{\ell}(\omega)\right]$ and $\left[\mathcal{Z}_{\ell}(\omega)\right]$ are $\left(N_{\ell} \times N_{\ell}\right)$ complex matrices, $\psi_{\ell}(\omega)$ is a $\left(N_{\ell} \times 1\right)$ complex vector, $\left[\mathcal{B}_{\ell}(\omega)\right]$ is a $\left(N_{\ell} \times N_{d}\right)$ complex matrix, and in which $\mathbf{c}^{+}$is a $\left(N_{d} \times 1\right)$ complex vector $\left(N_{\ell}\right.$ being the number of liner dofs and $N_{d}$ the number of cut-off excitation duct modes).

\section{B. Computational reduced-order model using a pellicular projection}

Following the explanations given at the beginning of Section III.A the uncertainties will be taken into account by using the nonparametric probabilistic approach [2]. Since there is a non negligible number of liner dofs, the static condensation only is not sufficient. Such an approach then requires the introduction of a reduced-order model associated with Eq (11). For such a construction we need to introduce a reduced-order basis that is chosen as a pellicular basis (see [19, 24, 25]).

This pellicular basis is associated with the acoustic radiation of the liner delimited by its boundary $\Gamma$ on which a thin acoustic layer (the pellicular domain) is introduced. Considering that the acoustic layer thickness is infinitely thin regarding to the acoustic wavelength, a set of acoustic modes related to the pellicular domain is computed solving the eigenvalue problem $\left[\mathcal{K}_{L}\right] \boldsymbol{\phi}_{\alpha}=\lambda_{\alpha}\left[\mathcal{M}_{L}\right] \phi_{\alpha}$ derived from the computational model, in which the symmetric $\left(N_{\ell} \times N_{\ell}\right)$ real matrices $\left[\mathcal{K}_{L}\right]$ and $\left[\mathcal{M}_{L}\right]$ are positive definite and represent, respectively, the acoustic thickness matrix and the associated mass matrix. The eigenvector $\phi_{\alpha}$ belonging to $\mathbb{R}^{N_{\ell}}$ is called a pellicular eigenvector and $\lambda_{\alpha}$ is its corresponding eigenvalue. These pellicular eigenvectors verify the classical orthogonal properties. Let $n_{\ell}$ be the number of pellicular eigenvectors $\phi_{1}, \ldots, \phi_{n_{\ell}}$ that are retained for the construction of the reduced-order model. We then introduce the $\left(N_{\ell} \times n_{\ell}\right)$ real matrix $[\Phi]$ whose columns are $\phi_{1}, \ldots, \phi_{n_{\ell}}$.

The computational reduced-order model is obtained by projecting Eq. [11] on the subspace spanned by [Ф] and is thus written as,

$$
\begin{gathered}
\psi_{\ell}(\omega)=[\Phi] \mathbf{q}(\omega), \\
([\mathbb{A}(\omega)]+[\mathbb{Z}(\omega)]) \mathbf{q}(\omega)=[\mathbb{B}(\omega)] \mathbf{c}^{+},
\end{gathered}
$$

in which $\mathbf{q}(\omega)$, which belongs to $\mathbb{C}^{n_{\ell}}$, is a complex vector of the generalized coordinates, and where the $\left(n_{\ell} \times n_{\ell}\right)$ complex matrix $[\mathbb{A}(\omega)]=[\Phi]^{T}\left[\mathcal{A}_{\ell}(\omega)\right][\Phi]$, the $\left(n_{\ell} \times n_{\ell}\right)$ complex matrix $[\mathbb{Z}(\omega)]=[\Phi]^{T}\left[Z_{\ell}(\omega)\right][\Phi]$, and the $\left(n_{\ell} \times N_{d}\right)$ complex matrix $[\mathbb{B}(\omega)]=[\Phi]^{T}\left[\mathcal{B}_{\ell}(\omega)\right]$.

In order to implement the nonparametric probabilistic approach, matrix $[\mathbb{A}(\omega)]$ and $[\mathbb{Z}(\omega)]$ need specific algebraic properties related to conservative and dissipative properties. For instance, the liner generalized impedance matrix $[\mathbb{Z}(\omega)]$ has to be decomposed in real, imaginary, symmetric and skew-symmetric parts in order to extract the exact term that is responsible for the dissipative in the computational model. Such algebraic properties and decompositions are not presented in this paper.

In the framework of the nonparametric probabilistic approach, the uncertainties in the computational model will be implemented by substituting complex matrices $[\mathbb{A}(\omega)]$ and $[\mathbb{Z}(\omega)]$ by random complex matrices $[\mathbf{A}(\omega)]$ and $[\mathbf{Z}(\omega)]$. The 
uncertainties on vector $\mathbf{c}^{+}$are taken into account by the parametric probabilistic, which consists in modeling $\mathbf{c}^{+}$by a random vector $\mathbf{C}^{+}$.

\section{Probabilistic model for uncertainty quantification}

As previously explained, three sources of uncertainties are taken into account. The first one is related to uncertainties induced by modeling errors in the impedance matrix of the liner appearing in the computational reduced-order model. The second one corresponds to model uncertainties in the aeroacoustic part of the computational model (including the mean-flow computation, the convected acoustic equations, and the duct acoustic related to the fan excitation). The third one is related to uncertainties in the acoustic excitation induced by the fan. The stochastic solver of the random equation is presented. For each value of the frequency, the Stochastic Reduced-Order Model (SROM) is solved by the Monte Carlo numerical simulation method [26]. The convergence analysis is carried out with respect to the number of realizations. For the first two sources of uncertainties, the nonparametric probabilistic approach is used, in particular the construction of the probabilistic model of the random matrices (see [2]). For the third one, the parametric probabilistic approach is used.

\section{A. Construction of the probabilistic model of the generalized liner impedance matrix}

In the nonparametric approach of uncertainties, complex matrix $[\mathbb{Z}(\omega)]$ introduced in Eq. (13) is then replaced by the random matrix $[\mathbf{Z}(\omega)]$, which exhibits a conservative part $\left[\mathbf{Z}^{\mathrm{NS}}(\omega)\right]$ and a dissipative part $\left[\mathbf{Z}_{I}^{\mathrm{S}}(\omega)\right]$ as,

$$
[\mathbf{Z}(\omega)]=\left[\mathbf{Z}^{\mathrm{NS}}(\omega)\right]+j\left[\mathbf{Z}_{I}^{\mathrm{S}}(\omega)\right]
$$

Both conservative and dissipative parts have a proper stochastic modeling that is briefly summarized in the following paragraphs.

Construction of the probabilistic model of random complex matrix $\left[Z^{\mathrm{NS}}(\omega)\right]$ (conservative part). The liner conservative impedance matrix $\left[\mathbf{Z}^{\mathrm{NS}}(\omega)\right]$ is constructed as follows by using the polar decomposition (cf Appendix X.A) of deterministic complex matrix $\left[\mathbb{Z}^{\mathrm{NS}}(\omega)\right]$ (assuming that $\left[\mathbb{Z}^{\mathrm{NS}}(\omega)\right]$ is invertible) as $\left[\mathbb{Z}^{\mathrm{NS}}(\omega)\right]=\left[U_{\mathbb{Z}}(\omega)\right]\left[T_{\mathbb{Z}}(\omega)\right]$ in which $\left[T_{\mathbb{Z}}(\omega)\right]$ is a positive-definite Hermitian $\left(n_{\ell} \times n_{\ell}\right)$ complex matrix and where $\left[U_{\mathbb{Z}}(\omega)\right]$ is a unitary complex $\left(n_{\ell} \times n_{\ell}\right)$ matrix . Then, using the appropriate stochastic modeling for each positive frequency $\omega$ fixed, the $\left(n_{\ell} \times n_{\ell}\right)$ complex random matrix $\left[\mathbf{Z}^{\mathrm{NS}}(\omega)\right]$ yields,

$$
\left[\mathbf{Z}^{\mathrm{NS}}(\omega)\right]=\left[U_{\mathbb{Z}}(\omega)\right]\left[L_{T}^{\mathbb{Z}}(\omega)\right]^{*}\left[\mathbf{G}_{\mathbb{Z}}^{\mathrm{NS}}\right]\left[L_{T}^{\mathbb{Z}}(\omega)\right],
$$

in which $\left[L_{T}^{\mathbb{Z}}(\omega)\right]$ is the result of the Cholesky factorization of the positive-definite Hermitian matrix $\left[T_{\mathbb{Z}}(\omega)\right]$. [ $\left.L_{T}^{\mathbb{Z}}(\omega)\right]$ is an upper triangular $\left(n_{\ell} \times n_{\ell}\right)$ complex matrix with positive diagonal. The random $\left(n_{\ell} \times n_{\ell}\right)$ real matrix $\left[\mathbf{G}_{\mathbb{Z}}^{\mathrm{NS}}\right]$ belongs to the set $\mathrm{SG}_{\varepsilon}^{+}$of random matrices defined in Appendix X.B in which the hyperparameter $\delta$ is denoted by $\delta_{\mathbb{Z}}$ allows for controling the level of uncertainties.

Construction of the probabilistic model of positive-definite symmetric real random matrix $\left[Z_{I}^{\mathrm{S}}(\omega)\right]($ dissipative part). The Cholesky factorization of the positive-definite symmetric real $\left(n_{\ell} \times n_{\ell}\right)$ matrix $\left[\mathbb{Z}_{I}^{S}(\omega)\right]$ is written as

$$
\left[\mathbb{Z}_{I}^{\mathrm{S}}(\omega)\right]=\left[L_{\mathbb{Z}}^{\mathrm{S}}(\omega)\right]^{T}\left[L_{\mathbb{Z}}^{\mathrm{S}}(\omega)\right]
$$

in which $\left[L_{\mathbb{Z}}^{\mathrm{S}}(\omega)\right]$ is an upper triangular $\left(n_{\ell} \times n_{\ell}\right)$ real matrix with positive diagonal. Let $\varepsilon>0$ be a fixed positive small parameter $(\varepsilon \ll 1)$. The random $\left(n_{\ell} \times n_{\ell}\right)$ complex matrix $\left[\mathbf{Z}_{I}^{\mathrm{S}}(\omega)\right]$ is then constructed as,

$$
\left[\mathbf{Z}_{I}^{\mathrm{S}}(\omega)\right]=\left[L_{\mathbb{Z}}^{\mathrm{S}}(\omega)\right]^{T}\left[\mathbf{G}_{\mathbb{Z}}^{\mathrm{S}}\right]\left[L_{\mathbb{Z}}^{\mathrm{S}}(\omega)\right],
$$

in which $\left[\mathbf{G}_{\mathbb{Z}}^{\mathrm{S}}\right]$ is a random $\left(n_{\ell} \times n_{\ell}\right)$ real matrix belonging to the set $\mathrm{SG}_{\mathcal{\varepsilon}}^{+}$defined and constructed in Appendix X.B in which the hyperparameter $\delta$ is denoted by $\delta_{\mathbb{Z}}$ and is the same as the one used for randomizing the matrix $\left[\mathbf{Z}^{\mathrm{NS}}(\omega)\right]$. 


\section{B. Construction of the probabilistic model of the generalized aeroacoustic matrix}

In order to construct the random aeroacoustic matrix $[\mathbf{A}(\omega)]$, we use the same methodology as the one used for the construction of the random matrix $\left[\mathbf{Z}^{\mathrm{NS}}(\omega)\right]$, such that,

$$
[\mathbf{A}(\omega)]=\left[U_{\mathbb{A}}(\omega)\right]\left[\mathbf{T}_{\mathbb{A}}(\omega)\right],
$$

in which $\left[U_{\mathbb{A}}(\omega)\right]$ is a unitary $\left(n_{\ell} \times n_{\ell}\right)$ complex matrix, such that $\left[U_{\mathbb{A}}(\omega)\right]^{*}\left[U_{\mathbb{A}}(\omega)\right]=\left[I_{n_{\ell}}\right]$, and where $\left[\mathbf{T}_{\mathbb{A}}(\omega)\right]$ is a positive-definite Hermitian $\left(n_{\ell} \times n_{\ell}\right)$ complex matrix whose construction is done as follows,

$$
\left[\mathbf{T}_{\mathbb{A}}(\omega)\right]=\left[L_{T}^{\mathbb{A}}(\omega)\right]^{*}\left[\mathbf{G}_{\mathbb{A}}\right]\left[L_{T}^{\mathbb{A}}(\omega)\right],
$$

in which $\left[L_{T}^{\mathbb{A}}(\omega)\right]$ corresponds to the Cholesky factorization of matrix $\left[T_{\mathbb{A}}(\omega)\right]$ and the random $\left(n_{\ell} \times n_{\ell}\right)$ real matrix $\left[\mathbf{G}_{\mathbb{A}}\right]$ belongs to the set $\mathrm{SG}_{\varepsilon}^{+}$defined in Appendix X.B. The hyperparameter $\delta_{\mathbb{A}}$ controls its level of uncertainties.

\section{Parametric probabilistic modeling of the fan acoustic excitation}

In order to take into account the uncertainties on the complex vector $\mathbf{c}^{+}$that has been introduced for describing the fan acoustic excitation, vector $\mathbf{c}^{+}$is modeled by a random vector $\mathbf{C}^{+}$with values in $\mathbb{C}^{N_{d}}$. This random vector $\mathbf{C}^{+}(\omega)$ depends on frequency $\omega$. Nevertheless, in order to simplify the notation, this frequency dependence will be removed in this section.

The methodology used for performing the probabilistic construction is based on the use of the Maximum Entropy principle. The first step consists in defining the nominal value of $\mathbf{c}^{+}$, the second one in defining the available information, and the third one in applying the Maximum Entropy principle under the constraints defined by the available information (see for instance [2], [27], [28], [29]). Details of the construction can be found in Appendix X.C

For $\alpha$ fixed in $\left\{1, \ldots, N_{d}\right\}$, the component $c_{\alpha}^{+}$of complex vector $\mathbf{c}^{+}=\left(c_{1}^{+}, \ldots, c_{N_{d}}^{+}\right)$, which corresponds to the nominal value is written as,

$$
c_{\alpha}^{+}=\underline{a}_{\alpha} e^{j \underline{\varphi}} \underline{\alpha}
$$

in which $\underline{a}_{\alpha}$ is the amplitude and $\underline{\varphi}_{\alpha}$ is the phase. The nominal value of complex vector $\mathbf{c}^{+}$is then represented by the real vector $\underline{\mathbf{a}}=\left(\underline{a}_{1}, \ldots, \underline{a}_{N_{d}}\right)$ of the nominal values of the amplitudes and the real vector $\underline{\varphi}=\left(\underline{\varphi}_{1}, \ldots, \underline{\varphi}_{N_{d}}\right)$ of the nominal values of the phases. The nominal values are assumed to be given and consequently, the deterministic real vectors $\underline{\mathbf{a}}$ and $\boldsymbol{\varphi}$ are given.

Then, the random vector $\mathbf{C}^{+}=\left(C_{1}^{+}, \ldots, C_{N_{d}}^{+}\right)$of component $\alpha$ is written as,

$$
C_{\alpha}^{+}=A_{\alpha}^{+} e^{j \Phi_{\alpha}^{+}} .
$$

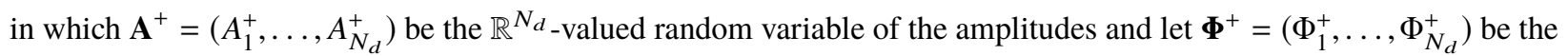

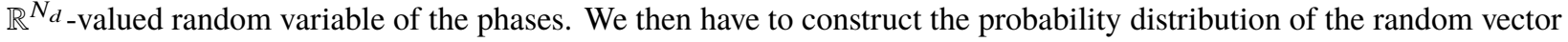
$\left(\mathbf{A}^{+}, \Phi^{+}\right)$using the Maximum Entropy principle for which the available information is defined in Appendix X.C

\section{Construction of the SROM by using the nonparametric probabilistic approach of uncertainties}

As previously explained, the nonparametric probabilistic approach of both the parametric uncertainties and the model uncertainties induced by modeling errors in the computational model of the acoustic radiation of an inlet consists in modeling matrix $[\mathbb{Z}(\omega)]$ defined by Eq. (13) by the random complex matrix $[\mathbf{Z}(\omega)]$ defined by Eq. (14) and $[\mathbb{A}(\omega)]$ defined by Eq. (13) by the random complex matrix $[\mathbf{A}(\omega)]$ defined by Eq. (18). The uncertainties on the fan acoustic excitation consist in modeling $\mathbf{c}^{+}(\omega)$ by the random complex vector $\mathbf{C}^{+}(\omega)$ as constructed in Section IV.C] The Quantity of Interest is a random vector $\mathbf{Q o I}(\omega)$ depending on $\omega$ that can be written as,

$$
\mathbf{Q o I}(\omega)=\left[\mathbb{F}_{\mathrm{QoI}}(\omega)\right] \mathbf{C}^{+}(\omega)-\left[\mathbb{A}_{\mathrm{QoI}}(\omega)\right] \mathbf{Q}(\omega),
$$

in which,

$$
\begin{gathered}
{\left[\mathbb{F}_{\mathrm{qoI}}(\omega)\right]=\left[\mathcal{O}_{\mathrm{obs}}(\omega)\right]\left[A_{a a}(\omega)\right]^{-1}\left[\mathcal{B}_{a}(\omega)\right],} \\
{\left[\mathbb{A}_{\mathrm{qoI}}(\omega)\right]=\left[\mathcal{O}_{\mathrm{obs}}(\omega)\right]\left[A_{a a}(\omega)\right]^{-1}\left[A_{a \ell}(\omega)\right][\Phi],}
\end{gathered}
$$


where $\left[\mathbb{O}_{\mathrm{obs}}(\omega)\right]$ is the observation matrix such that,

$$
\mathbf{q o I}(\omega)=\left[\bigcirc_{\mathrm{obs}}(\omega)\right] \psi_{a}(\omega) .
$$

The random vector $\mathbf{Q}(\omega)$ of the generalized coordinates verifies the random equation,

$$
([\mathbf{A}(\omega)]+[\mathbf{Z}(\omega)]) \mathbf{Q}(\omega)=[\mathbb{B}(\omega)] \mathbf{C}^{+}(\omega) .
$$

\section{E. Solving the stochastic matrix equation and constructing the confidence regions}

The Monte Carlo method is used for solving the SROM. It consists in computing a set of $v_{s}$ independent realizations, with $v_{s}$ sufficiently large, of the random quantities of interest by using Eqs. (22) and (26). From this set of independent realizations, estimates of the statistics for the quantities of interest are computed, such as probability density functions, moments (means, standard deviations), confidence intervals. The convergence of the estimates are analyzed with respect to $v_{s}$.

The frequency band of analysis $\left[\omega_{\min }, \omega_{\max }\right]$ is sampled in $n_{\omega}$ points $\left\{\omega_{1}, \ldots \omega_{n_{\omega}}\right\}$. For given $\omega_{j}, v_{s}$ realizations $\mathbf{Q o I}\left(\omega_{j} ; \theta_{\ell}\right)$ for $\ell=1, \ldots, v_{s}$ are given by

$$
\mathbf{Q o I}\left(\omega_{j} ; \theta_{\ell}\right)=\left[\mathbb{F}_{\mathrm{qoI}}\left(\omega_{j}\right)\right] \mathbf{C}^{+}\left(\omega_{j} ; \theta_{\ell}\right)-\left[\mathbb{A}_{\mathrm{qoI}}\left(\omega_{j}\right)\right] \mathbf{Q}\left(\omega_{j} ; \theta_{\ell}\right),
$$

in which the realization $\mathbf{Q}\left(\omega_{j} ; \theta_{\ell}\right)$ of random vector $\mathbf{Q}\left(\omega_{j}\right)$ is given by solving the deterministic linear matrix equation,

$$
\left(\left[\mathbf{A}\left(\omega_{j} ; \theta_{\ell}\right)\right]+\left[\mathbf{Z}\left(\omega_{j} ; \theta_{\ell}\right)\right]\right) \mathbf{Q}\left(\omega_{j} ; \theta_{\ell}\right)=\left[\mathbb{B}\left(\omega_{j}\right)\right] \mathbf{C}^{+}\left(\omega_{j} ; \theta_{\ell}\right),
$$

in which $\left\{\left[\mathbf{A}\left(\omega_{j} ; \theta_{\ell}\right)\right],\left[\mathbf{Z}\left(\omega_{j} ; \theta_{\ell}\right)\right], \mathbf{C}^{+}\left(\omega_{j} ; \theta_{\ell}\right), \ell=1, \ldots, v_{s}\right\}$ are $v_{s}$ independent realizations of random complex matrix $\left[\mathbf{A}\left(\omega_{j}\right)\right],\left[\mathbf{Z}\left(\omega_{j}\right)\right]$ and of random complex vector $\mathbf{C}^{+}\left(\omega_{j}\right)$.

Substituting $\mathbf{Q}\left(\omega_{j} ; \theta_{\ell}\right)$ given by Eq. 28] by Eq. 27] yields,

$$
\mathbf{Q o I}\left(\omega_{j} ; \theta_{\ell}\right)=\left[\mathbf{T}\left(\omega_{j} ; \theta_{\ell}\right)\right] \mathbf{C}^{+}\left(\omega_{j} ; \theta_{\ell}\right),
$$

in which,

$$
\left[\mathbf{T}\left(\omega_{j} ; \theta_{\ell}\right)\right]=\left[\mathbb{F}_{\mathrm{qoI}}\left(\omega_{j}\right)\right]-\left[\mathbb{A}_{\mathrm{qoI}}\left(\omega_{j}\right)\right]\left(\left[\mathbb{A}\left(\omega_{j}\right)\right]+\left[\mathbf{Z}\left(\omega_{j} ; \theta_{\ell}\right)\right]\right)^{-1}\left[\mathbb{B}\left(\omega_{j}\right)\right]
$$

is a complex matrix.

\section{Configuration and computational model of a generic industrial nacelle}

In this section, we present the computational model that is used to assess the performance of an acoustic liner in its operating environment.

In a first step, for a given flight condition, CFD is used for calculating velocity and mass density fields in the steady flow. In a second step, the computational aeroacoustic model is constructed.

For the aeroacoustic model including the liner, the finite/infinite element discretization shown in Figure 3 , is used. There are three fluid boundaries:

- the liner surfaces (red and orange surfaces), which allows for calculating the impedance of the liners as a function of the thermodynamic values on their surface, and for imposing it to the incident excitation pressure field.

- the fan excitation surface (green color), which allows for constructing the incident pressure field imposed to the system throught the duct.

- the near/far-field surface, which allows for imposing the continuity of the velocity potential between near-field and far-field.

Note that the existence of a system symmetry allows for using a half model for which a zero pressure condition (perfect reflection) is imposed on the symmetry plane. The system is then solved in two steps, one for the symmetric part (positive excitation azimuthal orders), and a second one for the anti-symmetric part (negative excitation azimuthal orders). There are 460000 dofs in the computational model of the half system for a maximum frequency of $1000 \mathrm{~Hz}$, 

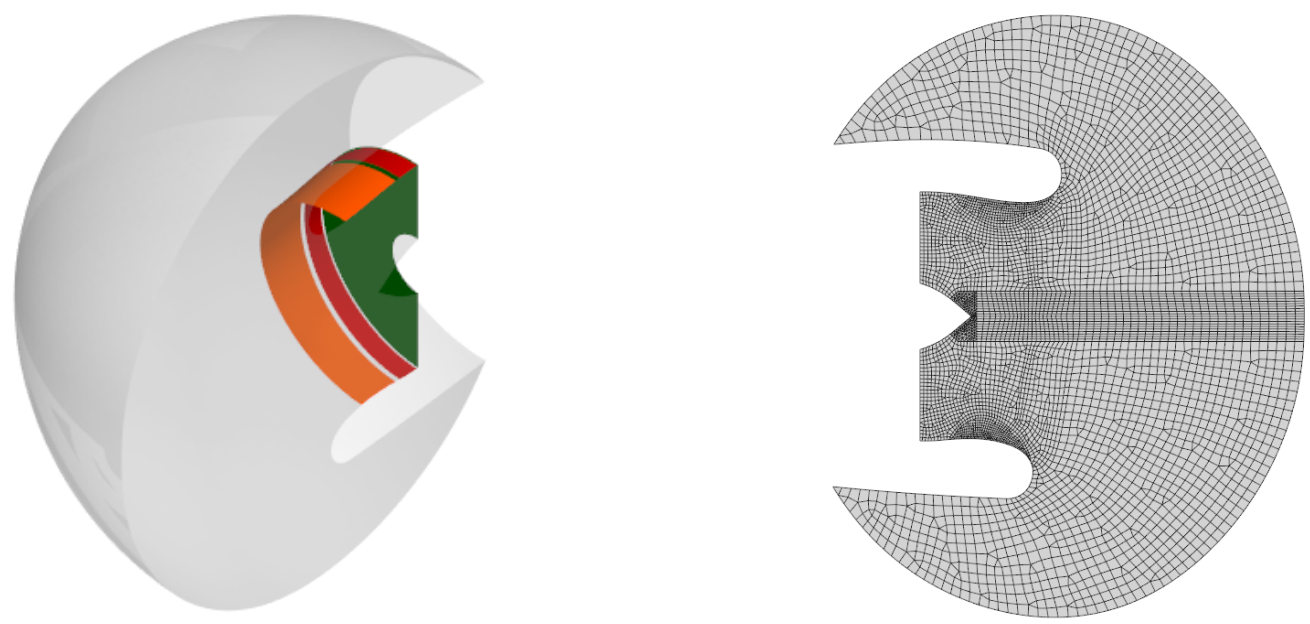

Fig. 3 Left : Finite/Infinite Element discretization of the half-3D generic nacelle intake (red: liner surface, green: fan excitation surface, purple: Infinite Elements). Right : 2D section of the half-3D generic aeroacoustic mesh shown on the left figure.

and 3000000 dofs for the $2000 \mathrm{~Hz}$ mesh. The quantities of interest are the far-field acoustic pressure that are captured by a microphone array located at $46 \mathrm{~m}$ of the nacelle axis, as it can be seen in Figure 4 , and ring acoustic pressure that are captured by a microphone array located on the nacelle intake lip. The fan acoustic contributions are observed and allows for computing both the far-field and the ring directivity modal SPL (Sound Pressure Level) quantities. The SPL broadband azimuthal quantity (far-field) consists in calculating the quadratic mean of the acoustic pressure over cut-on excitation modes, accounting for statistically independent random phases on $[0,2 \pi]$. Whereas, for the ring, azimuthal (or circumferential) SPL are computed using the phase of each microphone and the modal contribution of the fan excitation recovered on each microphone. In the present work, several flight conditions are studied, which correspond to acoustic
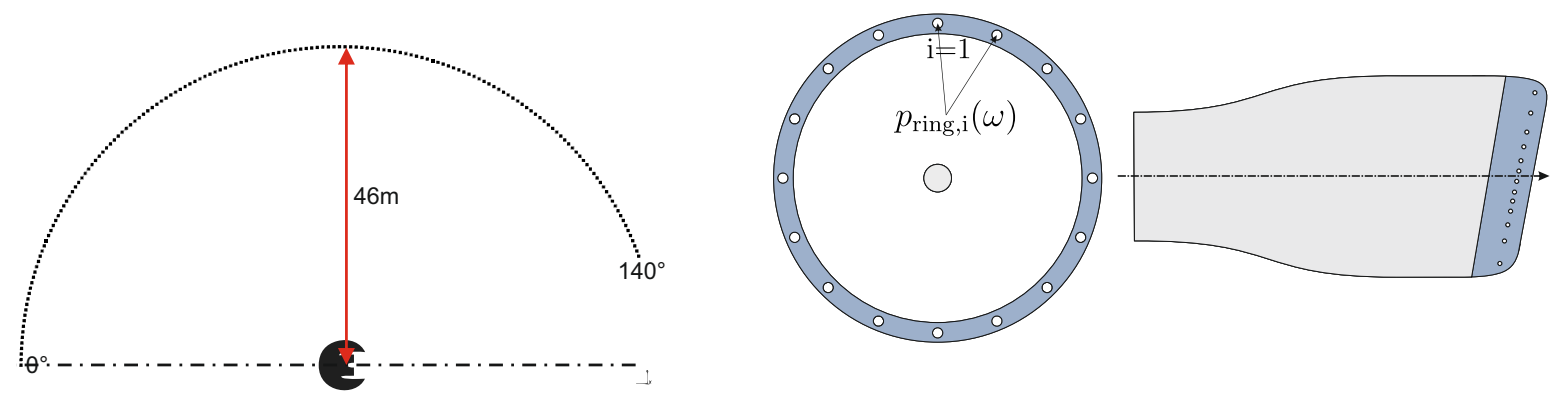

Fig. 4 Left: far-field microphone array. Right: ring microphone array located in the nacelle intake lip. certification points following ICAO rules [30].

\section{Definition of the Quantities of Interest}

\section{A. Quantities of Interest on the ring} Acoustic pressure on the ring

$$
p_{\text {ring }, \mathrm{i}}(\omega)=\sum_{m=-N_{\mathrm{azi}}}^{N_{\mathrm{azi}}} a_{m}(\omega) e^{j m \varphi_{i}},
$$

in which $\omega$ is the angular frequency, $m$ is the azimuthal order, $a_{m}(\omega)$ is the complex amplitude of the azimuthal order $m$, 
and $\varphi_{i}=2 \pi(i-1) / N_{\text {mics }}$ is the angle in $\mathrm{rad}$ of the $i^{\text {th }}$ microphone, in which $N_{\text {mics }}$ is the number of microphones on the ring. Equation (31) can be rewritten, in a matrix form, as

$$
\mathbf{p}_{\text {ring }}(\omega)=[\Phi] \mathbf{a}(\omega),
$$

in which $\mathbf{p}_{\text {ring }}(\omega)=\left(p_{\text {ring, }, 1}(\omega), \ldots, p_{\text {ring, } N_{\text {mics }}}\right)$ is the complex vector in $\mathbb{C}^{N_{\text {mics }}}, \mathbf{a}(\omega)=\left(a_{-N_{\text {azi }}}(\omega), \ldots, a_{0}(\omega), \ldots, a_{N_{\text {azi }}}(\omega)\right)$ is the complex amplitude vector in $\mathbb{C}^{2 N_{\text {azi }}+1}$, and $[\Phi]$ is the complex matrix in $\mathbb{M}_{N_{\text {mics }}, 2 N_{\text {azi }}+1}(\mathbb{C})$ such that $[\Phi]_{i m}=e^{j m \varphi_{i}}$.

Azimuthal amplitude for the ring acoustic pressure Assuming that $2 N_{\mathrm{azi}}+1 \leq N_{\text {mics }}$, the complex amplitude vector $\mathbf{a}(\omega)$ is obtained by using the left pseudo-inversion, as

$$
\mathbf{a}(\omega)=\left(\left([\Phi]^{*}[\Phi]\right)^{-1}[\Phi]^{*}\right) \mathbf{p}_{\text {ring }}(\omega) .
$$

Azimuthal Sound Pressure Level for the ring acoustic pressure The azimuthal amplitude of azimuthal order $m$ of the acoustic pressure on the ring, expressed in $\mathrm{dB}$, is defined, for $m \in\left\{-N_{\mathrm{azi}}, \ldots, 0, \ldots, N_{\mathrm{azi}}\right\}$, by

$$
\operatorname{SPL}_{\text {ring }, m}(\omega)=10 \log _{10}\left(\frac{\left|a_{m}(\omega)\right|^{2}}{p_{\text {ref }}^{2}}\right) .
$$

\section{B. Quantities of Interest for the far-field}

Acoustic pressure in the far-field For the $i^{\text {th }}$ microphone on the far-field arc defined in Fig. 4 for which there are $N_{\text {mics }}$ microphones, the acoustic pressure is denoted by $p_{\mathrm{ff}, i}(\omega)$. The complex vector in $\mathbb{C}^{N_{\text {mics }}}$ of the acoustic far-field pressures is then defined by

$$
\mathbf{p}_{\mathrm{fff}}(\omega)=\left(p_{\mathrm{ff}, 1}(\omega), \ldots, p_{\mathrm{ff}, N_{\text {mics }}}(\omega)\right) .
$$

Sound Pressure Level in the far-field The sound pressure level of the $i^{\text {th }}$ microphone related to $p_{\mathrm{ff}, \mathrm{i}}(\omega)$ (Eq. . 35 ) is defined by

$$
\operatorname{SPL}_{\mathrm{ff}, \mathrm{i}}(\omega)=10 \log _{10}\left(\frac{\left|p_{\mathrm{ff}, \mathrm{i}}(\omega)\right|^{2}}{p_{\text {ref }}^{2}}\right),
$$

\section{Definition of experimental QoIs}

Experimental Azimuthal Sound Pressure Level on the ring The experimental data are acquired on the ring that consists of $N_{\text {mics }}=100$ flush-mounted microphones distributed on the intake lip (see Figure 4). The azimuthal sound pressure level related to $a_{m}^{\exp }(\omega)$ is given by Eq. (33) is written on the ring(see Eq. (37)), as

$$
\mathrm{SPL}_{\text {ring, } m}^{\exp }(\omega)=10 \log _{10}\left(\frac{\left|a_{m}^{\exp }(\omega)\right|^{2}}{p_{\text {ref }}^{2}}\right),
$$

Experimental Sound Pressure Level in the far-field The sound pressure level in the far-field is written as

$$
\mathrm{SPL}_{\mathrm{ff}, \mathrm{i}}^{\exp }(\omega)=10 \log _{10}\left(\frac{\left.||_{\mathrm{ff}, \mathrm{i}}^{\exp }(\omega)\right|^{2}}{p_{\mathrm{ref}}^{2}}\right) .
$$




\section{Definition of simulated QoIs}

The stochastic computational aeroacoustic model used for representing the experimental set-up depicted in Figure 4p, is the one defined in section IV] Since the aeroacoustic computational model is linear, in order to avoid the call to the computational model a large number of times during the identification algorithm, the acoustic pressures on the ring and in the far-field, are firstly computed for a given reference modal intensity $I_{\alpha}^{+, \text {ref }}(\omega)$. For instance, such a reference $I_{\alpha}^{+, \text {ref }}(\omega)$ is chosen as $100 \mathrm{~dB}$ for all $\omega$ and for all $\alpha$. Then, these results are used for computing the acoustic pressures on the ring and in the far-field for a given modal intensity $I_{\alpha}^{+}(\omega)$. This is the reason why we need to rewrite the different QoIs in function of the modal intensity $I_{\alpha}^{+}$through the use of the reference modal intensity $I_{\alpha}^{+, \text {ref }}(\omega)$.

\section{A. Expression of the different Quantities of Interest as a function of the modal intensity $I_{\alpha}^{+}$}

The acoustic pressure $p_{i}(\omega)$ for each microphone $i$ (ring or far-field) and for mode $\alpha$ is computed using the computational aeroacoustic model. This acoustic pressure corresponds to an injected acoustic modal intensity $I_{\alpha}^{+ \text {,ref }}$ for each duct mode $\alpha$. The square of the acoustic pressure modulus corresponding to each duct mode $\alpha$ corresponding to $I_{\alpha}^{+ \text {,ref }}(\omega)$, is defined by

$$
\left|p_{\alpha}^{\mathrm{ref}}(\omega)\right|^{2}=p_{\mathrm{ref}}^{2} 10^{\left(I_{\alpha}^{+, \text {ref }}(\omega) / 10\right)} .
$$

For the modal intensity $I_{\alpha}^{+}(\omega)$, the square of the acoustic pressure modulus is then written as

$$
\left|p_{\alpha}(\omega)\right|^{2}=p_{\text {ref }}^{2} 10^{\left(I_{\alpha}^{+}(\omega) / 10\right)},
$$

which can be rewritten using the reference modal intensity $I_{\alpha}^{+, \text {ref }}$ as

$$
\left|p_{\alpha}(\omega)\right|^{2}=p_{\text {ref }}^{2} 10^{\left(I_{\alpha}^{+}(\omega)-I_{\alpha}^{+, \text {ref }}(\omega)\right) / 10},
$$

Expression of the simulated QoIs on the ring Let $\mathbf{a}^{\alpha}(\omega) \in \mathbb{C}^{2 N_{\text {azi }}+1}$ be the complex amplitude vector of mode $\alpha$ such that

$$
\mathbf{a}^{\alpha, \text { ref }}(\omega)=\left(\left([\Phi]^{*}[\Phi]\right)^{-1}[\Phi]^{*}\right) \mathbf{p}_{\text {ring }}^{\alpha, \text { ref }}(\omega),
$$

in which $\mathbf{p}_{\text {ring }}^{\alpha, \text { ref }}(\omega)$ is the vector in $\mathbb{C}^{N_{\text {mics }}}$ of the acoustic pressures on the ring computed for the reference modal intensity $I_{\alpha}^{+, \text {ref }}$. The azimuthal sound pressure level SPL $\mathrm{S}_{\text {ring, }, m}^{\text {sim }}(\omega)$ on the ring for the modal intensity $I_{\alpha}^{+}(\omega)$ is then given, for $m \in\left\{-N_{\text {azi }}, \ldots, 0, \ldots, N_{\text {azi }}\right\}$, by

$$
\mathrm{SPL}_{\text {ring }, m}^{\text {sim }}(\omega)=10 \log _{10}\left(\frac{1}{N_{d}} \sum_{\alpha=1}^{N_{d}} \frac{\left|a_{m}^{\alpha, \text { ref }}(\omega)\right|^{2}}{p_{\text {ref }}^{2}} \times 10^{\left(I_{\alpha}^{+}(\omega)-I_{\alpha}^{+, \text {ref }}(\omega)\right) / 10}\right) .
$$

Expression of the simulated far-field QoIs The sound pressure level in the far-field for the modal intensity $I_{\alpha}^{+}(\omega)$ is given by

$$
\operatorname{SPL}_{\mathrm{ff}}^{\operatorname{sim}}(\omega)=10 \log _{10}\left(\frac{1}{N_{d}} \sum_{\alpha=1}^{N_{d}} \frac{\left|p_{\mathrm{ff}}^{\alpha, \text { ref }}(\omega)\right|^{2}}{p_{\mathrm{ref}}^{2}} \times 10^{\left(I_{\alpha}^{+}(\omega)-I_{\alpha}^{+, \text {ref }}(\omega)\right) / 10}\right),
$$

in which $\mathbf{p}_{\mathrm{ff}}^{\alpha \text {,ref }}(\omega)$ is the vector in $\mathbb{C}^{N_{\text {mics }}}$ of the acoustic pressures on the far-field arc computed for the reference modal intensity $I_{\alpha}^{+, \text {ref }}$.

\section{B. Notation for the random variable and the mean value of the QoIs}

For the hardwall case (no liner), in the stochastic computational aeroacoustic model, the random quantities are (1) the modal intensity $I_{\alpha}^{+}(\omega)$, whose hyperparameters of the probabilistic model are $\underline{I}_{\alpha}^{+}(\omega)$ and $\sigma_{\alpha}$ (see Section VII.A), and (2) the generalized aeroacoustic matrix for which the hyperparameter is $\delta_{A}$. In this condition, the sound pressure level becomes random and will denote these random variables and their mean values as follows.

- The random azimuthal sound pressure level on the ring, defined by Eq. (43), is denoted $\operatorname{SPL}_{\text {ring, } m}^{\text {sim }}(\omega)$ (notation unchanged). A realization of this random variable is $\operatorname{SPL}_{\text {ring, } m}^{\text {sim }}(\omega ; \theta)$. Its mean value is denoted $\underline{\operatorname{SPL}}_{\text {ring, }, m}^{\text {sim }}(\omega)$. 
- The random sound pressure level in the far-field, defined by Eq. (44], is denoted $\operatorname{SPL}_{\mathrm{ff}}^{\mathrm{sim}}(\omega)$ (notation unchanged). A realization of this random variable is $\operatorname{SPL}_{\mathrm{ff}}^{\mathrm{sim}}(\omega ; \theta)$. Its mean value is denoted $\underline{\operatorname{SPL}}_{\mathrm{ff}}^{\text {sim }}(\omega)$.

\section{Sensitivity analysis with respect to uncertainties}

This section aims to present some results of the sensitivity analysis with respect to uncertainties in order to assess their impact on both far-field and ring modal pressure response.

\section{A. Sensitivity study of modal Sound Pressure Levels towards uncertainties}

In this subsection, the sensitivity of modal QoIs towards aeroacoustic and liner uncertainties on specific modes is undertaken. The main objective is to observe the raw impact of uncertainties on those modes, by avoiding unwanted effects such as the smoothing due to the averaging over all modes accounted for in the calculation of global SPLs. A comparison between hardwall configuration with aeroacoustic uncertainties only and lined configuration with liner uncertainties only is done as well as a comparison between far-field and ring datasets. This preliminary study is done for the same flight condition (approach) and frequency (1 BPF), and allows for controlling the validity of the problem physical representativeness. For this specific configuration, fan noise signature contains multimodal (broadband) noise and tonal noise. Tonal noise is generally carried by modes for which the azimuthal order corresponds to the engine order, and/or the rotor-stator interaction mode (Tyler and Sofrin [31]). As these specific modes are cut-on in this specific configuration, the same sensitivity analysis should be done on another configuration, for which the excitation modal content contains these modes. The present preliminary study is carried out for a relatively simple configuration that allows for computational costs to remain low (and thus allow for more analyses).

First mode $(m, n)=(0,1)$ The first mode generally radiates in the nacelle axis direction and is not impacted by lined surfaces since its acoustic energy is concentrated at the center. This mode is then interesting to be investigated in order to confirm that liner uncertainties will have low to zero impact on the acoustic response in this configuration.

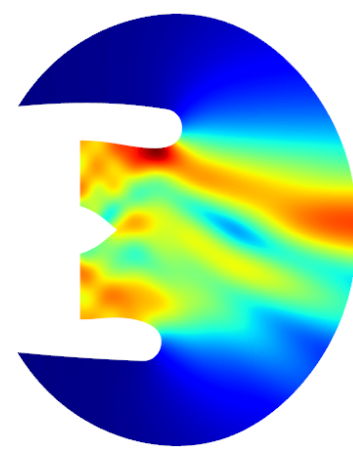

(a) Hardwall duct view

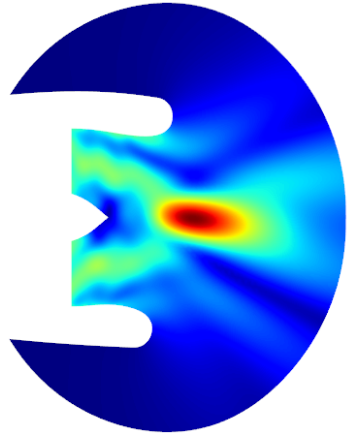

(b) Lined duct view

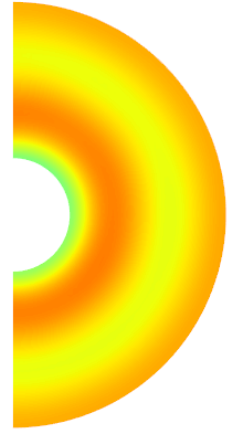

(c) Hardwall fan view

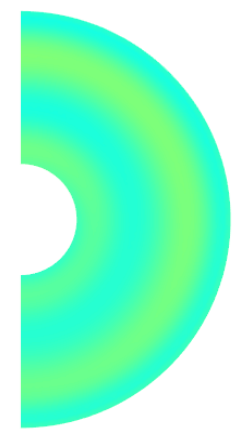

(d) Lined fan view

Fig. 5 Visualization of the acoustic pressure (norm) for the first mode $(m, n)=(0,1)$ inside the duct from two perspectives: side and front. Hardwall and lined configuration are depicted.

On Fig. 5, the acoustic pressure (norm) for the first mode $(m, n)=(0,1)$ is shown from two perspectives. Inside the duct (Fig. $5 \mathrm{a}$ and Fig. $5 \mathrm{~b}$, the acoustic pressure is quite heterogeneous along the nacelle axis, depicting a zone where it is higher (at the top, close to the lip for the hardwall configuration, and outside along the nacelle axis for the lined configuration). In a cross section of the nacelle where the fan is located (Fig. 5c and Fig. 5d), the difference between hardwall and lined configuration is not important (only the global amplitude of the swelling mode is different).

On Fig. 6, the sensitivity of the mode $(m, n)=(0,1)$ towards aeroacoustic and liner uncertainties is presented, observed on the ring microphone array. For the hardwall condition (Fig. 6a), one can notice that the confidence region associated with each one of the uncertainty level values (from 1 to $10 \%$ ) thicken then quickly stabilize. This is a saturation phenomenon that occurs for $\delta_{\mathrm{A}}=7.5 \%$, and means that by adding more uncertainties, the thickness of the confidence region would not increase much. This cannot be observed on the lined dataset for which uncertainties have a low impact on ring acoustic pressure (Fig. 6b, which is not surprising as the acoustic energy in the duct is located at the center. 


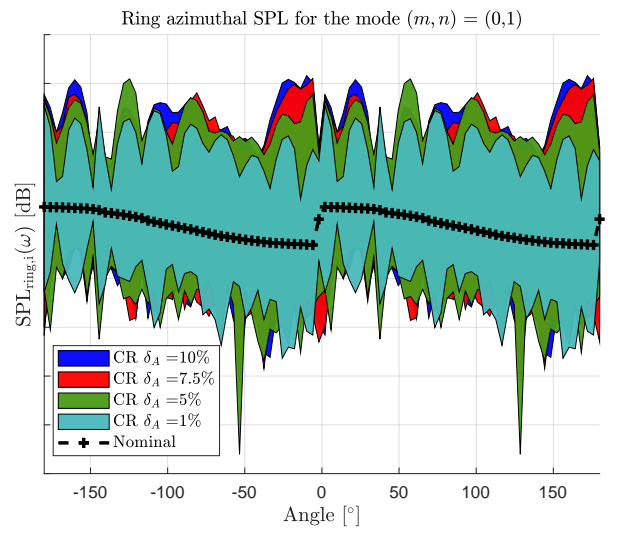

(a) Hardwall with aeroacoustic uncertainties only

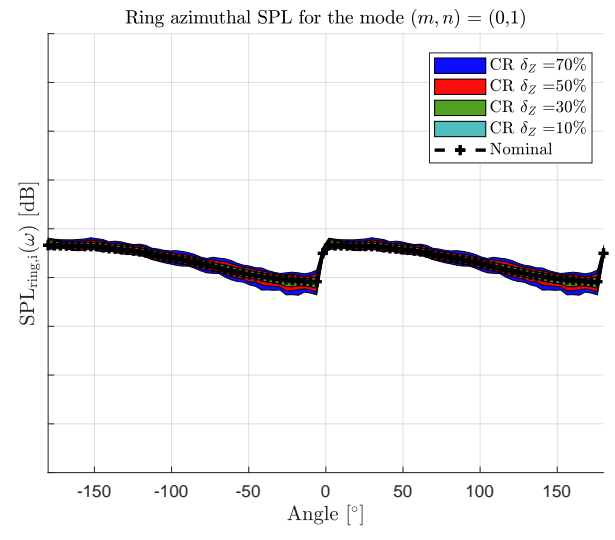

(b) Lined with liner uncertainties only

Fig. 6 Sensitivity of modal ring SPL data towards aeroacoustic uncertainty level $\delta_{\mathrm{A}}$ (left) and liner uncertainty level $\delta_{Z}$ (right) for the approach condition at the blade passing frequency $f=654 \mathrm{~Hz}(1 \mathrm{BPF})$ for the mode $(m, n)=(0,1)$ by comparison between nominal data (black line) and simulation data for different values of parameters $\delta_{\mathrm{A}}$ and $\delta_{\mathrm{Z}}$. One graduation equals $5 \mathrm{~dB}$.

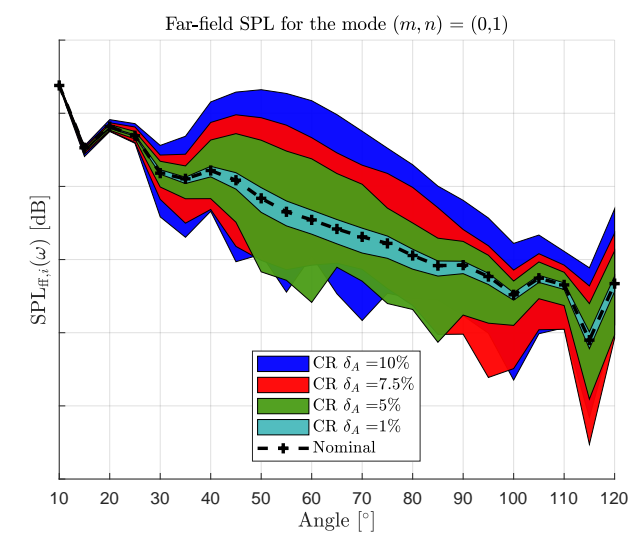

(a) Hardwall configuration with aeroacoustic uncertainties only

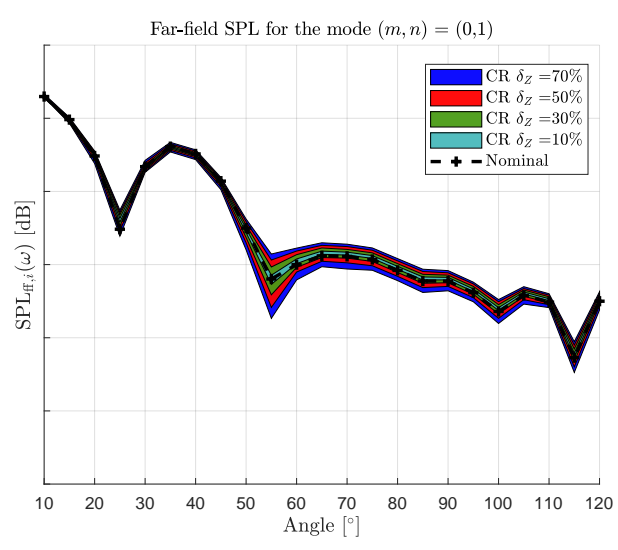

(b) Lined configuration with liner uncertainties only

Fig. 7 Sensitivity of far-field modal SPL data towards aeroacoustic uncertainty level $\delta_{\mathrm{A}}$ (left) and liner uncertainty level $\delta_{\mathrm{Z}}$ (right) for the approach condition at the blade passing frequency $f=654 \mathrm{~Hz}(1 \mathrm{BPF}$ ) for the mode $(m, n)=(0,1)$ by comparison between nominal data (black line) and simulation data for different values of parameters $\delta_{\mathrm{A}}$ and $\delta_{\mathrm{Z}}$. One graduation equals $20 \mathrm{~dB}$.

On Fig. 7a, the impact of aeroacoustic uncertainties over the hardwall far-field dataset is assessed for different levels. One can notice that the confidence regions increase linearly as $\delta_{\mathrm{A}}$ increases. The saturation effect observed on Fig. 6a might appear for a greater value of $\delta_{\mathrm{A}}$ on the far-field dataset. Giving the presence of a rather heterogeneous repartition of energy inside the duct, and especially close to the lip (see Fig. 5b), the impact of aeroacoustic uncertainties seems legitimate. For the lined configuration (Fig. 7b), the impact of liner uncertainties is far less important than aeroacoustic uncertainties on the hardwall case. Nevertheless, the impact is much more visible on the far-field array than on the ring array for this configuration (see Fig. 6b) simply because the dynamic is different on both figures, the confidence region being approximately $2 \mathrm{~dB}$ thick for both ring and far-field datasets.

Last mode $(m, n)=(10,1)$ The second mode to be investigated correspond to the last cut-off mode of the excitation modal content. This mode is interesting because of its energy repartition inside the duct, which is mainly concentrated close to the duct wall. As this mode is supposed to be strongly attenuated by lined surfaces, liner uncertainties should 
have an impact on the acoustic response.

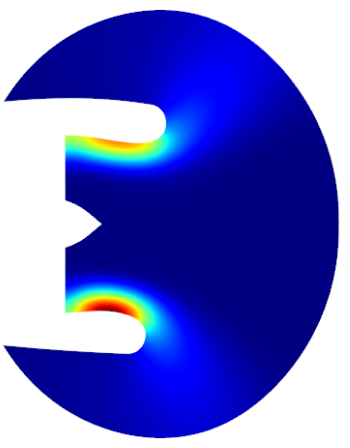

(a) Hardwall duct view

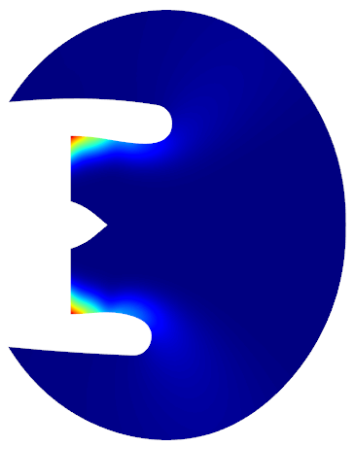

(b) Lined duct view

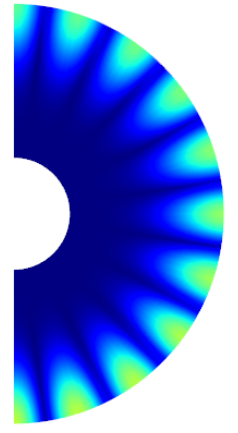

(c) Hardwall fan view

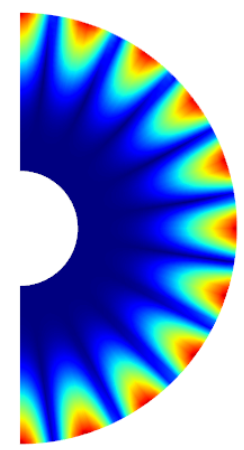

(d) Lined fan view

Fig. 8 Visualization of the acoustic pressure (norm) for the mode $(m, n)=(10,1)$ inside the duct from two perspectives: side and front. Hardwall and lined configuration are depicted.

Fig. 8 shows the acoustic pressure (norm) for the last mode $(m, n)=(10,1)$. Inside the duct (Fig. 8a), the acoustic pressure is mainly concentrated close to the duct wall. This is conform with what can be seen on Fig. $8 \mathrm{c}$, on which one can observe the alternation between pressure zeros and nonzeros. Since this mode has a high cut-off ratio and a short wavelength, and since its energy is concentrated on the wall, this mode is highly absorbed by liner surfaces. This is verified by a simple comparison between hardwall and lined duct views for which there is a clear impact of the liner on the acoustic pressure (Fig. 8a.

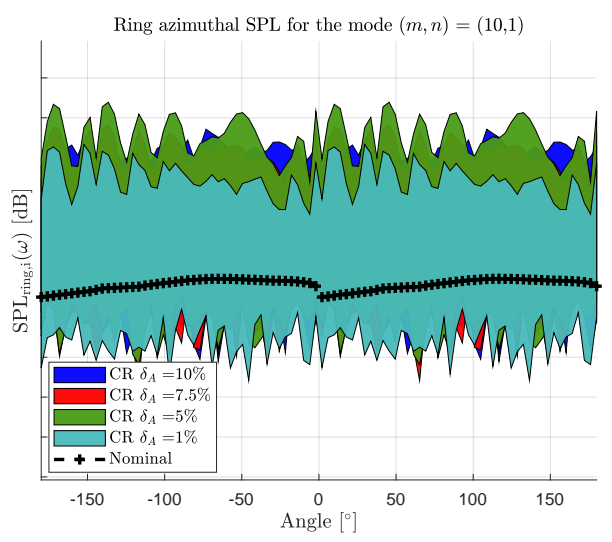

(a) Hardwall/aeroacoustic uncertainties only

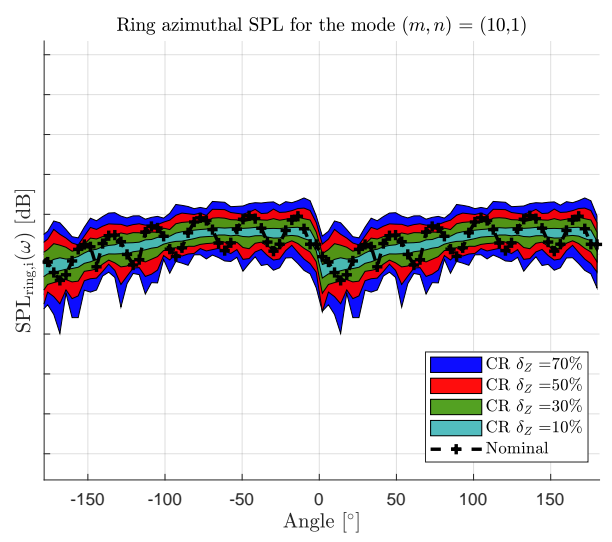

(b) Lined/liner uncertainties only

Fig. 9 Sensitivity of modal SPL data towards aeroacoustic uncertainty level $\delta_{\mathrm{A}}$ for the approach condition at the blade passing frequency $f=654 \mathrm{~Hz}(1 \mathrm{BPF})$ for the mode $(m, n)=(10,1)$ by comparison between nominal data (black line) and simulation data with aeroacoustic uncertainties for different values of parameters $\delta_{\mathrm{A}}$ and $\delta_{\mathrm{Z}}$. One graduation equals $5 \mathrm{~dB}$.

On Fig. 9a, the saturation phenomenon is still present and even more important than previously presented (Fig. 6a) for the first mode. For $\delta_{\mathrm{A}}=5 \%$, ring data are saturated by aeroacoustic uncertainties. This is not the case for the lined configuration for which liner uncertainties have a little impact on ring data. They have still more impact on this mode than for the mode $(m, n)=(0,1)$ (see Fig. 6b). Since this mode has a high cut-off frequency (in comparison with the mode $(m, n)=(0,1))$, and consequently a short wavelength, this mode is more impacted by the presence of the liner and so by liner uncertainties.

Fig. 10a depicts the impact of aeroacoustic uncertainties on the ring dataset corresponding to the hardwall configuration. The mode $(m, n)=(10,1)$ seems very sensitive towards aeroacoustic uncertainties as confidence regions tend to thicken and stochastic means increase as $\delta_{\mathrm{A}}$ increase. But contrarily to the first mode (see Fig. 7a), there is 

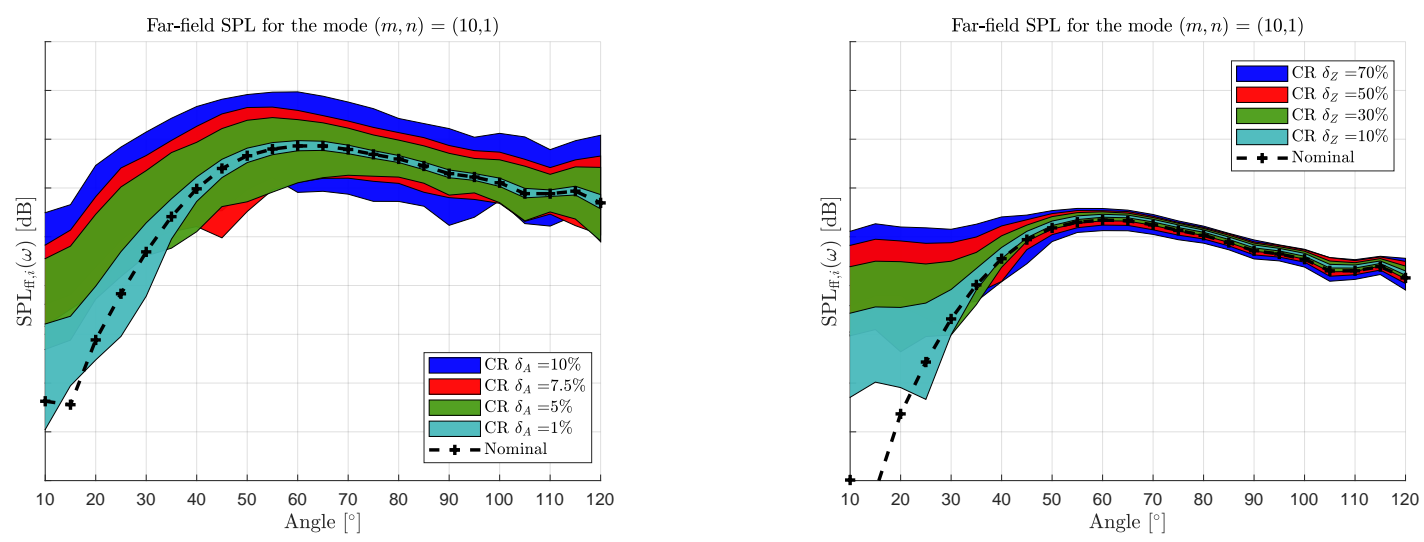

(a) Sensitivity of azimuthal ring modal SPL towards aeroa-(b) Sensitivity of broadband far-field modal SPL towards coustic uncertainties aeroacoustic uncertainties

Fig. 10 Sensitivity of modal SPL data towards aeroacoustic uncertainty level $\delta_{\mathrm{A}}$ and liner uncertainty level $\delta_{\mathrm{Z}}$ for the approach condition at the blade passing frequency $f=654 \mathrm{~Hz}(1 \mathrm{BPF})$ for the mode $(m, n)=(10,1)$ by comparison between nominal data (black line) and simulation data for different values of parameters $\delta_{\mathrm{A}}$ and $\delta_{\mathrm{Z}}$. One graduation equals $10 \mathrm{~dB}$.

not a clear angle range for which uncertainties have no impact. The same constat can be made for the lined case (Fig. $10 \mathrm{~b})$.

This study's results are not ground breaking but allow for verifying the validity of the method. The objective was to observe the impact of uncertainties over the ring dataset for two different modes whose energy repartition is opposite: one mode whose energy is concentrated around the nacelle axis, and another mode whose energy is located close to the wall. Aeroacoustic uncertainties have a strong impact on both modes, whereas liner uncertainties have impact only on the last mode, which is logical as it is strongly attenuated by liner surfaces.

\section{B. Sensitivity of global Sound Pressure Levels towards uncertainties}

In this subsection, global SPLs are used, as calculated using Eq. (43) for ring datasets, and Eq. (44) for far-field datasets.

Aeroacoustic uncertainties A first step consists in observing the impact of several aeroacoustic uncertainty levels ( $\delta_{\mathrm{A}}$ values) towards global SPLs (for both ring and far-field data). The range of $\delta_{\mathrm{A}}$ ranges from minimum to maximum intuitive values such as the problem physical meaning would be respected (for example $90 \%$ of aeroacoustic uncertainties is not a reasonable value).

On Fig. 11, the sensitivity study of global SPL towards aeroacoustic uncertainty level is presented for the approach condition at the BPF. $\delta_{\mathrm{A}}$ varies from $1 \%$ to $10 \%$. On the left figure (Fig. 11a), the impact of aeroacoustic uncertainties on the ring modal SPL is observed. One can notice that the sensitivity of $S P L_{\mathrm{ring}, m}$ is a logarithm function of $\delta_{\mathrm{A}}$. While, it seems that the sensitivity of the far-field SPL (broadband) is a linear function of $\delta_{\mathrm{A}}$, as it can be seen on Fig. $11 \mathrm{~b}$. This phenomenon has been encountered during the modal SPL sensitivity study (subsection VIII.A).

As explained, aeroacoustic uncertainties have a logarithmic impact on ring data, whereas a linear impact is observed on far-field data. This difference can be explained by several factor. As the aeroacoustic uncertainties increase, a saturation effect is quickly observed on ring data. While this saturation might eventually be obtained for a much higher value of $\delta_{\mathrm{A}}$ on far-field data (this is the case for $\delta_{\mathrm{A}}=50 \%$, see Fig. $11 \mathrm{c}$.

If we take the example of $\delta_{\mathrm{A}}=1 \%$, ring data are much more impacted than far-field data. The difference of the impact force between ring and far-field data is actually closely linked to the nature of the modes that are captured by the different microphones. This can be explained by the relative distance of the observer (ring or far-field) to the acoustic source (the fan). When duct modes exit the nacelle intake, they are captured by the ring, then propagate to be later captured by the far-field microphones. Indeed, one can notice that the ring, from its relatively close distance to the emission source (fan), is more likely to capture high cut-off frequency modes for which the acoustic wavelength is small. Whereas, as the far-field arc of microphone is far from the source, low cut-off frequency modes (which are strongly directive) will 


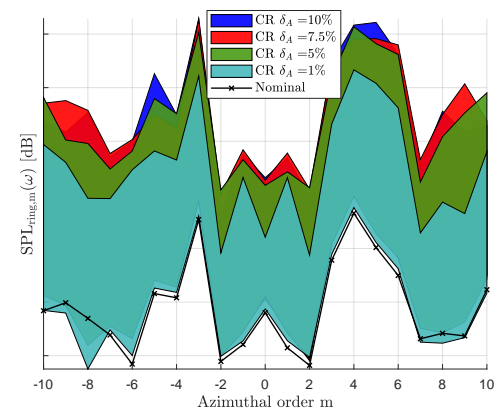

(a) Ring azimuthal SPL

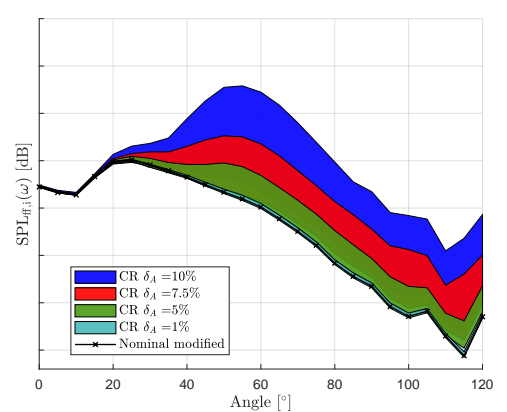

(b) Far-field SPL

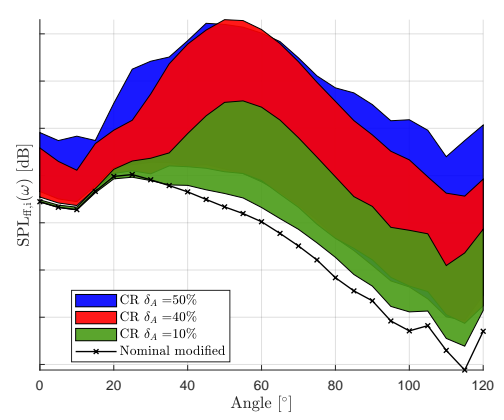

(c) Far-field SPL (saturation)

Fig. 11 Sensitivity of global SPL data towards aeroacoustic uncertainty level $\delta_{\mathrm{A}}$ for the approach condition at the blade passing frequency $f=654 \mathrm{~Hz}(1 \mathrm{BPF})$ by comparison between experimental data (black line) and simulation data with modified modal content and aeroacoustic uncertainties for several values. One graduation equals $5 \mathrm{~dB}$.

mainly be captured, while high cut-off frequency modes will already be attenuated. Also, because high cut-off ratio modes have less energy than low cut-off frequency modes, the impact of uncertainties is more likely to have a stronger impact on the former. This explains the difference observed for a low level of uncertainties.

Liner uncertainties A sensitivity study of global sound pressure levels for both ring and far-field stochastic data towards liner uncertainties is needed to observe how the uncertainty will impact the model. For this study, a variation of liner uncertainties is undertaken $\left(\delta_{\mathrm{Z}}=[10 \%, 30 \%, 50 \%, 70 \%]\right)$, keeping aeroacoustic uncertainties to zero. As it has been seen in subsection VIII.A liner uncertainties have a small impact towards modal SPL, for the ring as well as the far-field data. This could be explained by the fact that the liner studied is already optimized for this flight condition/frequency. In order to confirm or infirm this hypothesis, the global stochastic SPLs are also computed using a lined surface for which the admittance has been manually altered by a factor $200 \%$. Results can be seen on Fig. $12 \mathrm{~b}$.

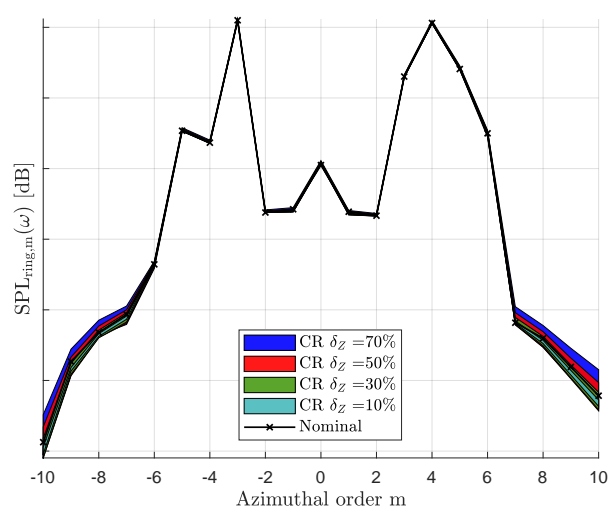

(a) Nominal impedance

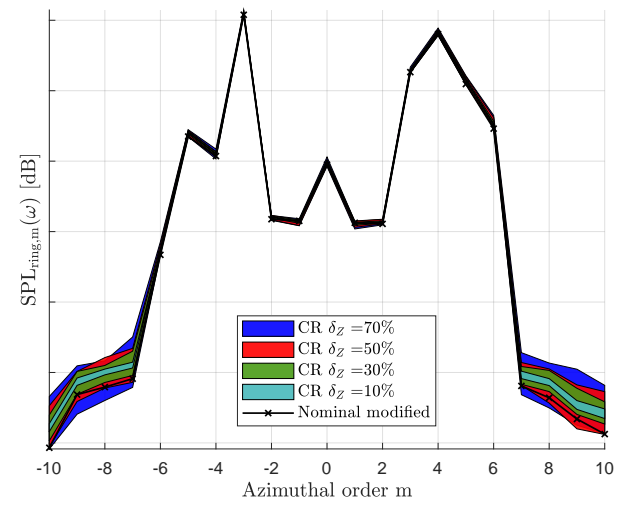

(b) Altered impedance

Fig. 12 Sensitivity of global Azimuthal SPL data towards liner uncertainty level $\delta_{Z}$ for the approach condition at the blade passing frequency $f=654 \mathrm{~Hz}(1 \mathrm{BPF})$ by comparison between experimental data (black line) and simulation data with modified modal content and aeroacoustic uncertainties for several values: $\delta_{\mathrm{A}}=10 \%$ (cyan confidence region), $\delta_{\mathrm{A}}=30 \%$ (green confidence region), $\delta_{\mathrm{A}}=50 \%$ (red confidence region), and $\delta_{\mathrm{A}}=70 \%$ (blue confidence region). Left: optimized admittance; Right: altered admittance by a factor $200 \%$. One graduation equals $5 \mathrm{~dB}$.

On Fig. 12 (both left and right figures), one can observe that lined uncertainties have a low impact on global ring data, especially for azimuthal orders in the range $[-6 ; 6]$. Whereas outside this range, the azimuthal SPL is slightly 
affected by liner uncertainties. As it has been extensively explained in the previous subsection, high cut-off frequency modes are more inclined to be affected by uncertainties since they are poorly energetic. The alteration of the admittance nevertheless implies a more important effectiveness of uncertainties, which is a good result in the framework of the liner optimization for which an optimum in terms of acoustic absorbtion as well as robustness is expected.

One can observe that on Fig. 12, the sensitivities towards liner uncertainties are slightly different when the liner admittance is not optimal. Altought this result is reassuring, it cannot be considered as conclusive. Liner uncertainties should have a more important impact on the detuned liner. Another study would consist in doing this analysis for a different flight condition and frequency for which the fan noise signature is more exhaustive.

\section{Inverse identification of uncertainty levels}

In this section, we present the results of the inverse identification of aeroacoustic, liner, and modal content uncertainty levels, which is the main objective of this paper. This study is divided into 4 main steps that are (1) the identification of the deterministic modal content $\underline{I}_{\alpha}$ using the hardwall configuration, (2) the identification of aeroacoustic uncertainties using the hardwall configuration, (3) the identification of liner uncertainties using the lined configuration and the previously identified aeroacoustic uncertainties, and (4) the identification of modal content uncertainty levels in presence of the previously identified aeroacoustic and liner uncertainty levels. For this purpose, the underneath objective is to try to frame experimental data with the $95 \%$ confidence region associated with the stochastic response on both ring and far-field arrays. We use global SPLs (see (44) and (43)), that have different sensitivities towards uncertainties. The identification could be simply done by using only far-field data but, as the modal content of experimental data is not known, we expect to reduce the error on the modal content by identifying one using different datasets.

\section{A. Identification of the mean modal content}

The lack of knowledge related to the acoustic excitation induced by the fan rotation is a major contribution to the overall uncertainty of the computational model. As explained in Section II.C.2 the acoustic excitation is represented by a finite sum of $N_{d}$ duct modes imposed to the acoustic system. In Section IV.C the deterministic modal intensity $\underline{I}_{\alpha}^{+}$ and its random counterpart $I_{\alpha}^{+}$of the duct modes have been defined. Concerning the construction of $I_{\alpha}^{+}$, an empirical

model based on civil-aircraft-manufacturer expertise is used. The current section aims to present the identification of the level of uncertainty associated with the acoustic excitation modal content. For that purpose, experimental data are used, corresponding to acoustic pressures measured at points located on a ring at the intake lip, and also on a far-field microphones array. The ring pressures are also simulated with the stochastic computational aeroacoustic model defined in Section IV The identification of the mean value $\underline{I}_{\alpha}^{+}$and the hyperparameter $\sigma_{\alpha}$ of the random variable $I_{\alpha}^{+}$is then performed by minimizing the distance between experimental and simulated data.

In this subsection, the deterministic modal content is identified by comparing experimental data and simulation data for which the modal content is modified, as explained in section II.C.2. The method consists in amplifying specific modes in order to lower the difference between simulation and experimental SPL quantities. For that purpose, the hardwall configuration is used (no liners).

Fig. 13 shows the results of the modal content optimization using the custom modal content specified in Section II.C.2 for which a broadband level, one positive azimuthal and one negative azimuthal emergence, and one emergence for the first mode are determined. The left figure (Fig. 13a) shows the azimuthal modified content associated with this optimization, in which emergences are shown. The right figure (Fig. 13b) shows the modification of the global sound pressure levels for both ring and far-field data (red line). The black line refers to the nominal calculation, without any modification of the modal content (all modes sent with a level of $100 \mathrm{~dB}$ ). The main goal here is to try to tune the modal content so as to reduce the difference between experimental data and simulated data to a minimum, for both ring and far-field data.

\section{B. Identification of the aeroacoustic uncertainty level}

Once the modal content is coarsely defined, the aeroacoustic uncertainties are identified by varying the parameter $\delta_{\mathrm{A}}$ from 0 to 1 . The main goal is to try to frame a maximum number of experimental points within the $95 \%$ confidence region plotted using superior and inferior quantiles.

As it has been showed in the previous paragraph VIII.B an aeroacoustic uncertainty level $\delta_{\mathrm{A}}=7.5 \%$ is sufficent to represent aeroacoustic uncertainties. In this paragraph, the associated results are shown for both ring and far-field global 


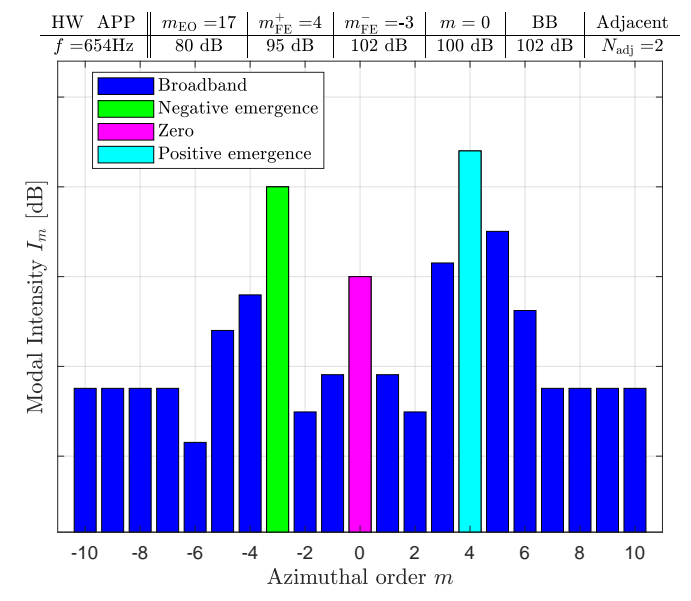

(a) Visualization of the excitation azimuthal content

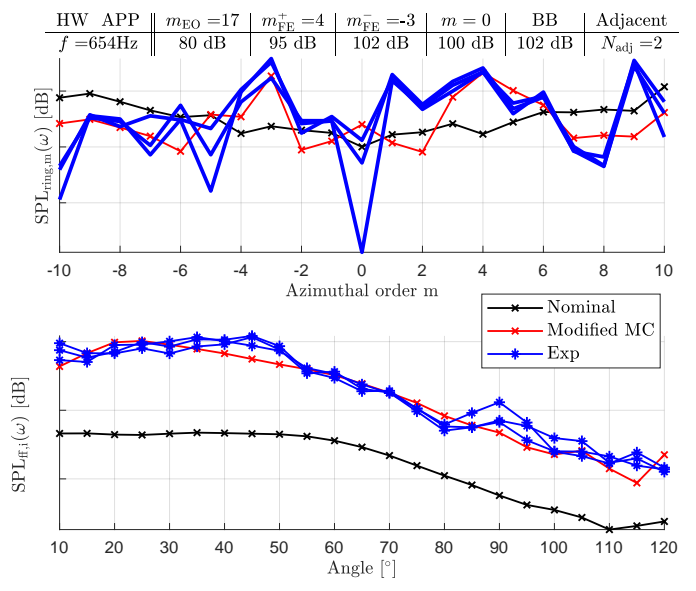

(b) Top: Ring data. Bottom: Far-field data

Fig. 13 Identification of the deterministic modified modal content for the approach condition at the blade passing frequency $f=654 \mathrm{~Hz}(1 \mathrm{BPF})$ by comparison between experimental data (blue line), nominal data (black line), and simulation data with modified modal content (red line). One graduation equals $10 \mathrm{~dB}$ for both plots.

SPLs.
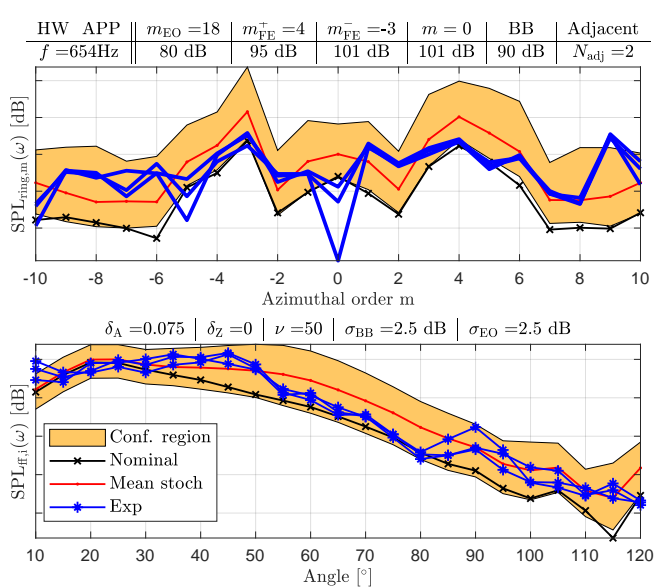

(a) Hardwall/Aeroacoustic uncertainties
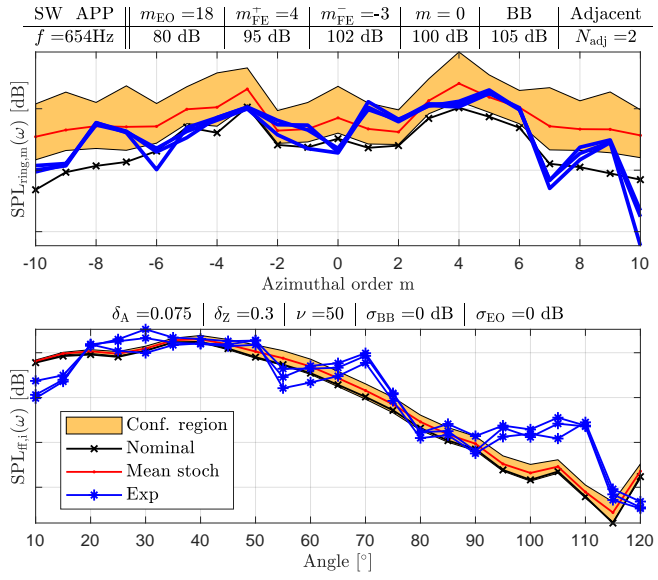

(b) Lined/Aeroacoustic and Liner uncertainties

Fig. 14 Left: Identification of the aeroacoustic uncertainty level $\delta_{\mathrm{A}}$ (one graduation equals $10 \mathrm{~dB}$ for top and $5 \mathrm{~dB}$ for bottom figure). Right: Identification of the liner uncertainty level $\delta_{Z}$. Approach condition at the blade passing frequency $f=654 \mathrm{~Hz}(1 \mathrm{BPF})$. Comparison between experimental data (blue line) and simulation data with modified modal content and aeroacoustic uncertainties (with the red line being the statistical mean, and the yellow patch the confidence region at 95\% (one graduation equals $20 \mathrm{~dB}$ for top and $5 \mathrm{~dB}$ for bottom figure). Top: Azimuthal SPL of ring data. Bottom: Broadband SPL of far-field data

On Fig. 14a global SPL for both ring and far-field data are computed for an aeroacoustic uncertainty level $\delta_{\mathrm{A}}=7.5 \%$. One can observe that for both configurations of microphones, the confidence region frames a satisfying number of experimental points, with the far-field being the most important target. Now that aeroacoustic uncertainties are coarsely identified, liner uncertainties can be added to the computational model in order to identify their level. 


\section{Identification of the liner uncertainty level}

As liner uncertainties are introduced, the computational model changes from hardwall to lined. Liner uncertainties are identified by varying the parameter $\delta_{\mathrm{Z}}$ from 0 to 1 . The main goal is to try and frame a maximum number of experimental points within the $95 \%$ confidence region plotted using superior and inferior quantiles. As it has been seen in the paragraph VIII.B. liner uncertainties have a relatively small impact on global SPL. The choice of their level is then difficult to make. We then intuitively decide to apply a reasonable level of liner uncertainty $\delta_{Z}=30 \%$.

On Fig. 14b, one can notice that the confidence region calculated using both aeroacoustic and liner uncertainties is not sufficiently large. By adding modal content uncertainties, the confidence region should thicken.

\section{Identification of the modal content uncertainty level}

As aeroacoustic and liner uncertainties has been fixed, the last parameters that allow for thickening the confidence region and frame a maximum of experimental points are the modal content uncertainties. Those are identified by varying the parameters $\sigma_{\mathrm{EO}}$ and $\sigma_{\mathrm{BB}}$ from 0 to $10 \mathrm{~dB}$, keeping a confidence region as thin as possible.
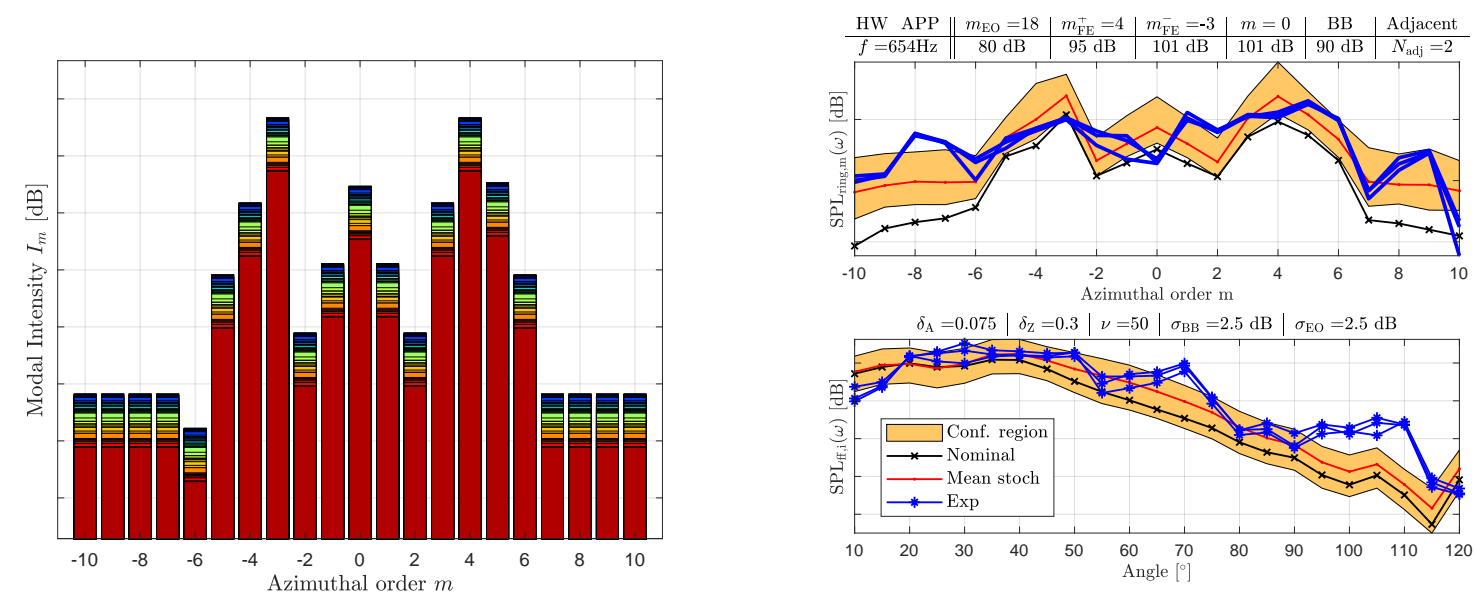

(a) Visualization of the excitation stochastic azimuthal con-(b) Top: Azimuthal SPL of ring data. Bottom: Broadband tent

SPL of far-field data

Fig. 15 Identification of the modal content uncertainty levels $\sigma_{\mathrm{EO}}$ and $\sigma_{\mathrm{BB}}$ for the approach condition at the blade passing frequency $f=654 \mathrm{~Hz}(1 \mathrm{BPF})$ by comparison between experimental data (blue line) and simulation data with modified modal content and aeroacoustic uncertainties (the red line being the statistical mean, and the yellow patch the confidence region) at $95 \%$. One graduation equals $20 \mathrm{~dB}$ for top and $5 \mathrm{~dB}$ for bottom figure.

On Fig. 15a, the azimuthal stochastic content is depicted, on which the variability on each azimuthal order is observed ( $\pm 2.5 \mathrm{~dB}$ for broadband modes and $\pm 2.5 \mathrm{~dB}$ for emergences). On Fig. $15 \mathrm{~b}$, the global ring and far-field SPL are plotted, taking into account the modified modal content (Subsection IX.A), aeroacoustic uncertainties (Subsection IX.B), liner uncertainties (Subsection IX.C), and modal content uncertainties. A finer optimization is then needed to obtain a better identification, by varying all parameters on small ranges around their previously identified values.

\section{E. Confirmation of the method validity for a different flight condition: cutback condition at 1 BPF}

As the liner is to be optimized for several engine speed (e.g flight conditions), it is important to validate the identification method for at least one other engine speed.

Fig. 16 presents the inverse identification of all uncertainty levels in the stochastic model of acoustic propagation for the cutback flight condition at $1 \mathrm{BPF}$. Qualitatively, the inverse identification can be considered as correctly done. There is a sufficient number of experimental points inside the confidence region for both far-field and ring datasets. An important thing to notice is that the far-field 40 to $60^{\circ}$ angle region is very sensitive towards aeroacoustic uncertainties and grows accordingly. This phenomenon is difficult to compensate with the modified modal content (which is logarithmic by essence). therefore, aeroacoustic uncertainties have to be kept relatively low to avoid the growing of energy in this specific angle range. That is why, for this example, modal content uncertainties are set to a high value, in order to 


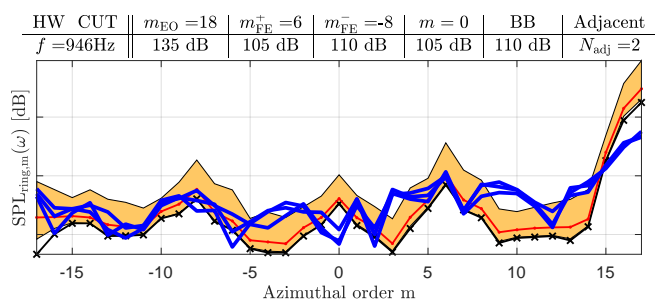

$\delta_{\mathrm{A}}=0.02\left|\delta_{\mathrm{Z}}=0\right| \nu=50\left|\sigma_{\mathrm{BB}}=0 \mathrm{~dB}\right| \sigma_{\mathrm{EO}}=0 \mathrm{~dB}$

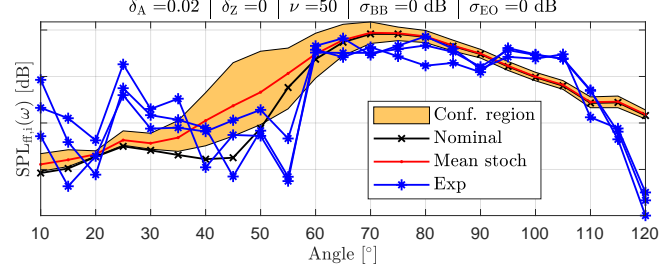

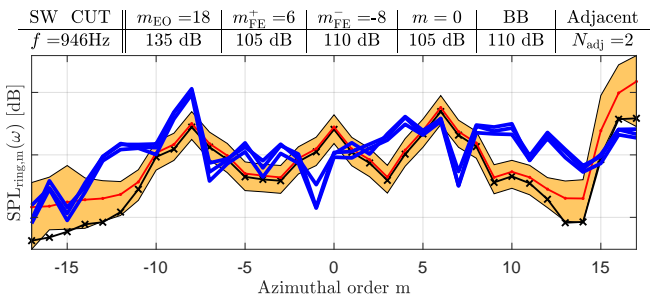

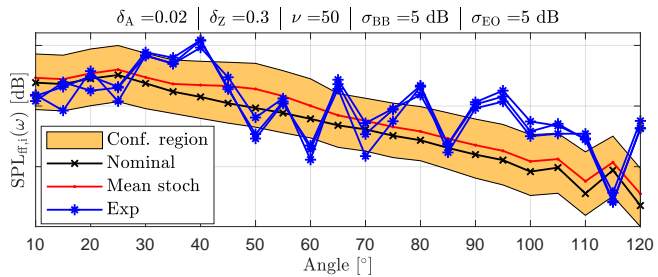

(a) Visualization of the excitation stochastic azimuthal con-(b) Top: Azimuthal SPL of ring data. Bottom: Broadband tent

SPL of far-field data

Fig. 16 Identification of all the uncertainty levels using relative QoIs for the cutback condition at the blade passing frequency $f=946 \mathrm{~Hz}(1 \mathrm{BPF})$ by comparison between experimental data (blue line) and simulation data with modified modal content, aeroacoustic, and liner uncertainties (the red line being the statistical mean, and the yellow patch the confidence region) at $95 \%$. Left: one graduation equals $20 \mathrm{~dB}$ for top and $5 \mathrm{~dB}$ for bottom figure. Right: one graduation equals $20 \mathrm{~dB}$ for top and $10 \mathrm{~dB}$ for bottom figure.

increase the confidence region thickness and frame a maximum number of experimental points. 


\section{Conclusion}

The main objective of the present study is the robust design of nacelle liners. As liner design is frozen in early phases of the aircraft development, the maturity of data is still very low. The idea is to anticipate changes in aircraft data that would impact the liner optimum design. For that purpose, uncertainties related to modeling errors and parameters errors in the computational model for liner performance have to be accounted for. In the computational model, the main sources of uncertainties correspond to the accounting of the flow (computed using potential Euler equations), the liner impedance calculation, and the fan acoustic excitation.

In this paper, we present the inverse identification of the deterministic modal content, the aeroacoustic, and liner uncertainty levels by using experimental data issued from a static ground test for different configurations. One fully hardwall and one fully treated, as explained in section $\mathrm{V}$ Also, several flight conditions (engine regimes corresponding to approach and cutback) and one frequency (1 BPF) are studied. From these configurations, two datasets are used, one close to the fan (on the nacelle intake lip) and one in the far-field. The main idea is to try to tune the modal content, its uncertainty levels as well as aeroacoustic and liner uncertainty levels by decreasing the distance between experimental and simulated ring data. Because ring microphones are evenly distributed in the circumferential direction, the azimuthal content should be easy to determine, whereas the radial content remains unknown. Then, the previously identified modal content and uncertainty levels (using ring data) are supposed to be coherent with far-field data. This means that, identifying the aforementioned parameters using only ring data should be sufficiently precise to also frame far-field experimental points.

As it has been observed for several applications presented here, the identification is not that simple. If the inverse identification of each parameter was a simple convex optimization problem with only one possible optimum, then, identifying parameters using ring data would be sufficient. But, this is not the case here, for there is a consequent number of parameters to optimize simultaneously. For example, there are several modal content definitions that can be used, each one giving different results when used on far-field data. The azimuthal "location" of emergences can whether have a strong impact on the far-field representation, or simply none, and the same is observed for the impact of uncertainty levels. This usually leads to severe differences between a tuning that is well-adapted to ring data, and a tuning that is well-adapted to far-field data, which are more important in the end. A compromise between the two is then to be made. In simpler terms, the precision of the identification of uncertainty levels strongly depends on the modal content knowledge.

The present study is preliminary and is mainly dedicated to the validation of the methodology, in addition to the identification of uncertainty levels. The respect of simple physical phenomena such as the impact of uncertainties over duct modes whose energy repartition is well defined is of major importance here. Then, the sensitivity of global sound pressure levels towards uncertainties is also assessed and allows for understanding the behavior of the stochastic modeling in an optimization framework. Above all, the synergy between the modal content and the sources of uncertainties is studied and allows for identifying the levels of uncertainties and the nominal modal content, as they are needed for the robust optimization of liners.

Nevertheless, considering the lack of knowledge related to the fan excitation modal content, which is of major importance, the identification of uncertainty levels, which has been presented here, can now be used for the robust optimization, in which for each set of design parameters, a Monte Carlo analysis is done (for which the uncertainty levels identified in this paper are fixed). The robust optimization then gives access to the optimum of the liner in terms of acoustic performance (absorption) and also in terms of robustness, which is related to the level of deviation of stochastic acoustic pressures. 


\section{Appendix}

A. Polar decomposition of a complex matrix

It is assumed that the $\left(n_{\ell} \times n_{\ell}\right)$ complex matrix $[\mathbb{Q}(\omega)]$ is invertible. Therefore, $[\mathbb{Q}(\omega)]$ can be written (polar decomposition) as,

$$
[\mathbb{Q}(\omega)]=\left[U_{\mathbb{Q}}(\omega)\right]\left[T_{\mathbb{Q}}(\omega)\right],
$$

in which $\left[U_{\mathbb{Q}}(\omega)\right]$ is a unitary $\left(n_{\ell} \times n_{\ell}\right)$ complex matrix, such that,

$$
\left[U_{\mathbb{Q}}(\omega)\right]^{*}\left[U_{\mathbb{Q}}(\omega)\right]=\left[I_{n_{\ell}}\right],
$$

in which $\left[U_{\mathbb{Q}}(\omega)\right]^{*}={\overline{\left[U_{\mathbb{Q}}(\omega)\right]}}^{T}$ is the transpose conjugate of matrix $\left[U_{\mathbb{Q}}(\omega)\right]$ and where $\left[I_{n_{\ell}}\right]$ is the $\left(n_{\ell} \times n_{\ell}\right)$ identity matrix. The matrix $\left[T_{\mathbb{Q}}(\omega)\right]$ belongs to the set $\mathbb{M}_{n_{\ell}}^{+}(\mathbb{C})$ of all the positive-definite Hermitian $\left(n_{\ell} \times n_{\ell}\right)$ complex matrices. This means that $\left[T_{\mathbb{Q}}(\omega)\right]$ is invertible, is such that $\left[T_{\mathbb{Q}}(\omega)\right]^{*}=\left[T_{\mathbb{Q}}(\omega)\right]$ (Hermitian symmetry), and is such that $\mathbf{z}^{*}\left[T_{\mathbb{Q}}(\omega)\right] \mathbf{z}>0$ for all $\mathbf{z}$ in $\mathbb{C}^{n_{\ell}}$ such that $\|\mathbf{z}\| \neq 0$ where $\|\mathbf{z}\|$ is the Hermitian norm in $\mathbb{C}^{n_{\ell}}$.

The construction of the representation defined by Eq. (45) can be done as follows. As $[\mathbb{Q}(\omega)]$ is assumed to be invertible, then the matrix $\left[H_{\mathbb{Q}}(\omega)\right]$ can be written as,

$$
\left[H_{\mathbb{Q}}(\omega)\right]=[\mathbb{Q}(\omega)]^{*}[\mathbb{Q}(\omega)],
$$

which belongs to $\mathbb{M}_{n_{\ell}}^{+}(\mathbb{C})$ and consequently, its spectral decomposition can be written as

$$
\left[H_{\mathbb{Q}}(\omega)\right]=\left[\Phi_{\mathbb{Q}}(\omega)\right]\left[S_{\mathbb{Q}}(\omega)\right]\left[\Phi_{\mathbb{Q}}(\omega)\right]^{*},
$$

in which $\left[S_{\mathbb{Q}}(\omega)\right]$ is the diagonal $\left(n_{\ell} \times n_{\ell}\right)$ real matrix of the positive eigenvalues of $[H(\omega)]$ and where $\left[\Phi_{\mathbb{Q}}(\omega)\right]$ is the $\left(n_{\ell} \times n_{\ell}\right)$ complex matrix of the eigenvectors such that $\left[\Phi_{\mathbb{Q}}(\omega)\right]^{*}[\Phi(\omega)]=\left[\Phi_{\mathbb{Q}}(\omega)\right]\left[\Phi_{\mathbb{Q}}(\omega)\right]^{*}=\left[I_{n_{\ell}}\right]$. Note that diagonal matrix $\left[S_{\mathbb{Q}}(\omega)\right]$ can also be viewed as the diagonal matrix of the singular values of complex matrix $[\mathbb{Q}(\omega)]$. Let us define the matrix $\left[T_{\mathbb{Q}}(\omega)\right]$ by

$$
\left[T_{\mathbb{Q}}(\omega)\right]=\left[\Phi_{\mathbb{Q}}(\omega)\right]\left[S_{\mathbb{Q}}(\omega)\right]^{1 / 2}\left[\Phi_{\mathbb{Q}}(\omega)\right]^{*} .
$$

It can be seen that $\left[T_{\mathbb{Q}}(\omega)\right]$ belongs to $\mathbb{M}_{n_{\ell}}^{+}(\mathbb{C})$. Let $\left[U_{\mathbb{Q}}(\omega)\right]$ be the complex matrix in $\mathbb{M}_{n_{\ell}}(\mathbb{C})$ defined (see Eq. 445 ) by,

$$
\left[U_{\mathbb{Q}}(\omega)\right]=[\mathbb{Q}(\omega)]\left[T_{\mathbb{Q}}(\omega)\right]^{-1} .
$$

Therefore, it can be verified that $\left[U_{\mathbb{Q}}(\omega)\right]^{*}\left[U_{\mathbb{Q}}(\omega)\right]=\left[I_{n_{\ell}}\right]$.

\section{B. Definition and construction of the set $\mathrm{SG}_{\varepsilon}^{+}$of random matrices [2]}

In this section, all the matrices are independent of frequency parameter $\omega$. A random $\left(n_{\ell} \times n_{\ell}\right)$ real matrix [G] belonging to $\mathrm{SG}_{\varepsilon}^{+}$is a random matrix with values in $\mathbb{M}_{n_{\ell}}^{+}(\mathbb{R})$, which is written as,

$$
[\mathbf{G}]=\frac{1}{1+\varepsilon}\left\{\left[\mathbf{G}_{0}\right]+\varepsilon\left[I_{n_{\ell}}\right]\right\},
$$

in which $\left[\mathbf{G}_{0}\right]$ is a random $\left(n_{\ell} \times n_{\ell}\right)$ real matrix with values in $\mathbb{M}_{n_{\ell}}^{+}(\mathbb{R})$, defined on $(\Theta, \mathcal{T}, \mathcal{P})$, which belongs to the set $\mathrm{SG}_{0}^{+}$of random matrices defined in [2]. Random matrix $\left[\mathbf{G}_{0}\right]$ has been constructed using the Maximum Entropy principle under the constraints defined by the following available information,

$$
E\left\{\left[\mathbf{G}_{0}\right]\right\}=\left[I_{n_{\ell}}\right] \quad, \quad E\left\{\log \left(\operatorname{det}\left[\mathbf{G}_{0}\right]\right)\right\}=v_{G_{0}} .
$$

in which $v_{G_{0}}$ is any constant such that $\left|v_{G_{0}}\right|<+\infty$. The probability distribution obtained of $\left[\mathbf{G}_{0}\right]$ is not Gaussian, depends on constant $v_{G_{0}}$, and is reparameterized using the dispersion parameter $\delta$, defined by,

$$
\delta=\left\{\frac{E\left\{\left\|\left[\mathbf{G}_{0}\right]-E\left\{\left[\mathbf{G}_{0}\right]\right\}\right\|_{F}^{2}\right\}}{\left\|E\left\{\left[\mathbf{G}_{0}\right]\right\}\right\|_{F}^{2}}\right\}^{1 / 2}=\left\{\frac{1}{n_{\ell}} E\left\{\left\|\left[\mathbf{G}_{0}\right]-\left[I_{n_{\ell}}\right]\right\|_{F}^{2}\right\}^{1 / 2} .\right.
$$


Consequently, the probability distribution of the non-Gaussian random matrix $\left[\mathbf{G}_{0}\right]$ depends only on one hyperparameter that is $\delta$. This hyperparameter allows for controlling the level of statistical fluctuations, that is to say, for controlling the level of uncertainties. Using $E\left\{\left[\mathbf{G}_{0}\right]\right\}=\left[I_{n_{\ell}}\right]$, Eq. [51) yields $E\{[\mathbf{G}]\}=\left[I_{n_{\ell}}\right]$. For $\theta$ fixed in $\Theta$, the realization $\left[\mathbf{G}_{0}(\theta)\right]$ is computed by using the following representation of random matrix $\left[\mathbf{G}_{0}\right]$,

$$
\left[\mathbf{G}_{0}\right]=[\mathbf{L}]^{T}[\mathbf{L}]
$$

in which $[\mathbf{L}]$ is an upper triangular random matrix with values in $\mathbb{M}_{n_{\ell}}(\mathbb{R})$ such that,

1) the random variables $\left\{[\mathbf{L}]_{j j^{\prime}}, j \leq j^{\prime}\right\}$ are mutually independent.

2) for $j<j^{\prime}$, we have $[\mathbf{L}]_{j j^{\prime}}=\sigma \mathcal{G}_{j j^{\prime}}$ in which $\sigma=\delta\left(n_{\ell}+1\right)^{-1 / 2}$ and where $\mathcal{G}_{j j^{\prime}}$ is a real-valued Gaussian random variable with zero mean and with a variance that is equal to 1 .

3) for $j=j^{\prime}$, we have $[\mathbf{L}]_{j j}=\sigma \sqrt{2 \mathcal{V}_{j}}$ where $\mathcal{V}_{j}$ is a positive-valued Gamma random variable whose probability density function with respect to $d v$ is written as

$$
p_{\mathcal{V}_{j}}(v)=1_{\mathbb{R}^{+}}(v) \frac{1}{\Gamma\left(\frac{n_{\ell}+1}{2(\delta)^{2}}+\frac{1-j}{2}\right)} v^{\frac{n_{\ell}+1}{2(\delta)^{2}}-\frac{1+j}{2}} e^{-v} .
$$

The random variable $\mathcal{V}_{j}$ can be rewritten as the nonlinear transformation $h\left(\mathcal{G}_{j j} ; n_{\ell} ; \delta\right)$ of a real-valued Gaussian random variable $\mathcal{G}_{j j}$ with zero mean and with a variance that is equal to 1 .

4) The Gaussian random variables $\left\{\mathcal{G}_{j j^{\prime}}, 1 \leq j \leq j^{\prime} \leq n_{\ell}\right\}$ are statistically independent.

Remarks.

1) The algebraic representation defined by Eq. (54) shows that although the entries $\left\{[\mathbf{L}]_{j j^{\prime}}, j \leq j^{\prime}\right\}$ of $[\mathbf{L}]$ are mutually independent, the entries $\left\{\left[\mathbf{G}_{0}\right]_{j j^{\prime}}, j \leq j^{\prime}\right\}$ of $\left[\mathbf{G}_{0}\right]$ are mutually dependent.

2) The diagonal entries $[\mathbf{L}]_{j j}, j=1, \ldots, n_{\ell}$ of random matrix $[\mathbf{L}]$ depend on $j$.

\section{Parametric probabilistic modeling of the fan acoustic excitation}

The methodology used for performing the probabilistic construction is based on the use of the Maximum Entropy principle. The first step consists in defining the nominal value of $\mathbf{c}^{+}$, the second one in defining the available information, and the third one in applying the Maximum Entropy principle under the constraints defined by the available information (see for instance [2], [27], [28], [29]).

(i) Nominal value. For $\alpha$ fixed in $\left\{1, \ldots, N_{d}\right\}$, the component $c_{\alpha}^{+}$of complex vector $\mathbf{c}^{+}=\left(c_{1}^{+}, \ldots, c_{N_{d}}^{+}\right)$is written as,

$$
c_{\alpha}^{+}=\underline{a}_{\alpha} e^{j \varphi} \underline{\varphi}_{\alpha}
$$

in which $\underline{a}_{\alpha}$ is the amplitude and $\underline{\varphi}_{\alpha}$ is the phase. The nominal value of complex vector $\mathbf{c}^{+}$is then represented by the real vector $\underline{\mathbf{a}}=\left(\underline{a}_{1}, \ldots, \underline{a}_{N_{d}}\right)$ of the nominal values of the amplitudes and the real vector $\underline{\varphi}=\left(\underline{\varphi}_{1}, \ldots, \underline{\varphi}_{N_{d}}\right)$ of the nominal values of the phases. The nominal values are assumed to be given and consequently, the deterministic real vectors $\underline{\mathbf{a}}$ and $\underline{\varphi}$ are given.

(ii) Available information. The component $\alpha$ of the complex random vector $\mathbf{C}^{+}=\left(C_{1}^{+}, \ldots, C_{N_{d}}^{+}\right)$is written as,

$$
C_{\alpha}^{+}=A_{\alpha}^{+} e^{j \Phi_{\alpha}^{+}} .
$$

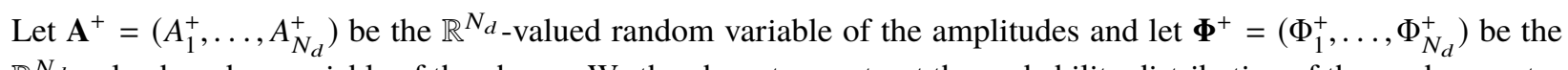

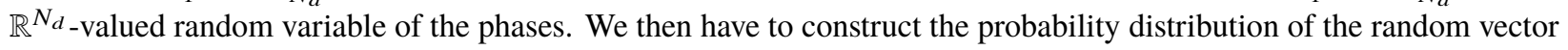
$\left(\mathbf{A}^{+}, \Phi^{+}\right)$using the Maximum Entropy principle for which the available information is defined as follows.

For $\alpha$ fixed in $\left\{1, \ldots, N_{d}\right\}$,

1) $A_{\alpha}^{+}$is a second-order random variable with values in $\mathbb{R}^{+}$.

2) The mean value of $A_{\alpha}^{+}$is $\underline{a}_{\alpha}$, that is to say, $E\left\{A_{\alpha}^{+}\right\}=\underline{a}_{\alpha}$.

3) For $a \rightarrow 0_{+}\left(0_{+}\right.$denotes 0 by upper values), the probability density function $a \mapsto p_{A_{\alpha}^{+}}(a)$ must go to zero (if not, the value of $p_{A_{\alpha}^{+}}(0)$ should be given and we have no information about this). The weak constraint that allows for imposing this condition is $E\left\{\log \left(A_{\alpha}^{+}\right)\right\}=b_{\alpha}^{+}$in which $\left|b_{\alpha}^{+}\right|<+\infty$ and where $\log$ is a Neperian logarithm. This unknown constant $b_{\alpha}^{+}$will be reexpressed in terms of the coefficient of variation $\delta_{\alpha}=\sigma_{\alpha} / \underline{a}_{\alpha}$ of random variable 
$A_{\alpha}^{+}$in which $\sigma_{\alpha}$ is the standard deviation of $A_{\alpha}^{+}$. This coefficient of variation is unknown and is, either used as a sensitivity parameter with respect to the level of uncertaities, or identified if experimental results are available.

4) The random variable $\Phi_{\alpha}^{+}$is with values in $\left[\varphi_{\alpha}-\pi, \varphi_{\alpha}+\pi\right]$.

5) $\Phi_{\alpha}^{+}$is therefore a second-order random variable and it is assumed that $E\left\{\Phi_{\alpha}^{+}\right\}=\underline{\varphi}_{\alpha}$.

(iii) Maximum Entropy principle. We have to construct the probability density function $(\mathbf{a}, \boldsymbol{\varphi}) \mapsto p_{\mathbf{A}^{+}, \boldsymbol{\Phi}^{+}}(\mathbf{a}, \boldsymbol{\varphi})$ of random variable $\left(\mathbf{A}^{+}, \boldsymbol{\Phi}^{+}\right)$, which is defined on $\left\{\prod_{\alpha=1}^{N_{d}} \mathbb{R}^{+}\right\} \times\left\{\prod_{\alpha=1}^{N_{d}}\left[\underline{\varphi}_{\alpha}-\pi, \underline{\varphi}_{\alpha}+\pi\right]\right\}$. Applying the Maximum Entropy principle under the constraints defined by (ii)-1 to (ii)-5 yields the following results ([2]).

1) The random variables $A_{1}^{+}, \ldots, A_{N_{d}}^{+}, \Phi_{1}^{+}, \ldots, \Phi_{N_{d}}^{+}$are statistically independent, which means that

$$
p_{\mathbf{A}^{+}, \boldsymbol{\Phi}^{+}}(\mathbf{a}, \varphi)=\prod_{\alpha=1}^{N_{d}}\left\{p_{A_{\alpha}^{+}}\left(a_{\alpha}\right) \times p_{\Phi_{\alpha}^{+}}\left(\varphi_{\alpha}\right)\right\},
$$

in which $\mathbf{a}=\left(a_{1}, \ldots, a_{N_{d}}\right)$ and $\boldsymbol{\varphi}=\left(\varphi_{1}, \ldots, \varphi_{N_{d}}\right)$. It should be noted that this result is due to the fact that no information is given concerning the dependencies of the components of random variables $\mathbf{A}^{+}$and $\mathbf{\Phi}^{+}$.

2) For $\alpha$ in $\left\{1, \ldots, N_{d}\right\}, A_{\alpha}^{+}$is a Gamma random variable for which its probability density function is written as

$$
p_{A_{\alpha}^{+}}\left(a_{\alpha}\right)=1_{] 0,+\infty[}\left(a_{\alpha}\right) \frac{\left(\delta_{\alpha}^{-2}\right)^{\delta_{\alpha}^{-2}}}{\Gamma\left(\delta_{\alpha}^{-2}\right) \underline{a}_{\alpha}}\left(\frac{a_{\alpha}}{\underline{a}_{\alpha}}\right)^{\delta_{\alpha}^{-2}-1} \exp \left\{-\frac{a_{\alpha}}{\delta_{\alpha}^{2} \underline{a}_{\alpha}}\right\},
$$

in which $0 \leq \delta_{\alpha}<1 / \sqrt{2}$ and where $\Gamma(\beta)$ is the Gamma function. The random variable $\Phi_{\alpha}^{+}$is uniform and its probability density function is written as

$$
p_{\Phi_{\alpha}^{+}}\left(\varphi_{\alpha}\right)=\frac{1}{2 \pi} 1_{\left[\underline{\varphi}_{\alpha}-\pi, \underline{\varphi}_{\alpha}+\pi\right]}\left(\varphi_{\alpha}\right) .
$$

Taking into account Eq. (60), random variable $\Phi_{\alpha}^{+}$can be written as

$$
\Phi_{\alpha}^{+}=\underline{\varphi}_{\alpha}+\varepsilon_{\alpha} \pi\left(2 U_{\alpha}^{+}-1\right),
$$

in which $U_{\alpha}^{+}$is a uniform random variable on $[0,1]$ and where we have introduced the indicator $\varepsilon_{\alpha}$ whose value is zero or one. This parameter allows for killing the statistical fluctuations of the phase taking $\varepsilon_{\alpha}=0$; if not $\varepsilon_{\alpha}=1$. (iv) Remarks concerning the use of the probabilistic model. For $\alpha$ fixed in $\left\{1, \ldots, N_{d}\right\}$,

1) if $\delta_{\alpha}=0$, then the amplitude $A_{\alpha}^{+}=\underline{a}_{\alpha}$ is deterministic and equal to the nominal value. In this case, $C_{\alpha}^{+}=\underline{a}_{\alpha} e^{j \Phi_{\alpha}^{+}}$ is a random complex coefficient with deterministic modulus and random phase. In addition, if $\varepsilon_{\alpha}=0$, then $C_{\alpha}^{+}=c_{\alpha}^{+}$is a complex deterministic coefficient equal to the nominal value.

2) if $\underline{a}_{\alpha}=0$, then $A_{\alpha}^{+}=0$ because a gamma random variable with zero mean is equal to zero. In this case, the complex random coefficient $C_{\alpha}^{+}$is zero.

3) if $\underline{a}_{\alpha}>0$, if $\delta_{\alpha}>0$, and if $\varepsilon_{\alpha}=0$, then the random complex coefficient $C_{\alpha}^{+}$is written as $C_{\alpha}^{+}=A_{\alpha}^{+} e^{j \underline{\varphi}}$. That is to say, the amplitude is random and the phase is deterministic.

Definition of the modal intensity $I_{\alpha}^{+}$In the present paragraph, the frequency $\omega$ is fixed and is removed for simplifying the writing. The acoustic pressure $p$ is related to the velocity potential $\varphi$ by Eq. (2) and the continuity condition $\psi_{\mathrm{i}}=\varphi$ on $\Gamma_{\mathrm{f}}$. The velocity potential $\varphi$, can be rewritten as

$$
\varphi=\varphi^{+}+\varphi^{-}
$$

where

$$
\varphi^{+}(x, y, z)=\sum_{\alpha} c_{\alpha}^{+} \varphi_{\alpha}(x, y) e^{j k_{z \alpha} z} .
$$

We then define $p_{\alpha}^{+}$, using Eq. (??), by

$$
p_{\alpha}^{+}=-j \omega \rho_{0} \varphi_{\alpha}^{+}-\rho_{0} \mathbf{v}_{0} \cdot \nabla \varphi_{\alpha}^{+} .
$$

in which $\varphi_{\alpha}^{+}$is the contribution of mode $\alpha$ in $\varphi^{+}$. The modal intensity $I_{\alpha}^{+}$, related to pressure $p_{\alpha}^{+}$, is defined by 


$$
I_{\alpha}^{+}=10 \log _{10}\left(\frac{\left|p_{\alpha}^{+}\right|^{2}}{p_{\text {ref }}^{2}}\right),
$$

in which $p_{\text {ref }}$ is a reference pressure.

Probabilistic model of $I_{\alpha}^{+}$The probabilistic model $I_{\alpha}^{+}$of the random modal intensity could be derived from the one used for $C_{\alpha}^{+}$. Nevertheless, for civil aircraft applications, a deterministic model $\underline{I}_{\alpha}^{+}$is often used by the manufacturers and this model has to be accounted for in the construction of the probabilistic model. This probabilistic model is thus constructed using $\underline{I}_{\alpha}^{+}$, which is chosen as the mean value of $I_{\alpha}^{+}$. The available information concerning the positive random variable $I_{\alpha}^{+}$is its mean value $\underline{I}_{\alpha}^{+}$and the amplitude of variations defined by a positive interval $\mathcal{I}_{\alpha} \subset \mathbb{R}^{+}$depending on $\alpha$ and containing the mean value. In addition, there is no available information concerning the statistical dependence of the random variables $I_{1}^{+}, \ldots, I_{N_{d}}^{+}$. Using the MaxEnt principle of Information Theory, it is concluded that the random variables $I_{1}^{+}, \ldots, I_{N_{d}}^{+}$are statistically independent, and the probability distribution of each $I_{\alpha}^{+}$is uniform with support $\mathcal{I}_{\alpha}$ and with mean value $\underline{I}_{\alpha}^{+}$. Interval $\mathcal{I}_{\alpha}$ is parameterized using a positive hyperparameter $\sigma_{\alpha}>0$ such that $\mathcal{I}_{\alpha}=\left[\underline{I}_{\alpha}^{+}\left(1-\sigma_{\alpha}\right), \underline{I}_{\alpha}^{+}\left(1+\sigma_{\alpha}\right)\right]$. Therefore, the random variable $I_{\alpha}^{+}$can be written as

$$
I_{\alpha}^{+}=\underline{I}_{\alpha}^{+}\left(1+\sigma_{\alpha} U_{\alpha}\right) \quad, \quad 0<\sigma_{\alpha}<1,
$$

in which $U_{\alpha}$ is a centered uniform random variable on the interval $[-1,1]$.

\section{References}

[1] Mohring, W., "A well posed acoustic analogy based on a moving acoustic medium," arXiv preprint arXiv:1009.3766, 2010.

[2] Soize, C., Uncertainty Quantification: An Accelerated Course with Advanced Applications in Computational Engineering, Vol. 47, Springer, 2017.

[3] Robinson, J., and Watson, W., "Performance of a checkerboard liner with uncertain impedances," 11th AIAA/CEAS Aeroacoustics Conference, 2005, p. 2850.

[4] Jones, M., Parrott, T., and Watson, W., "Uncertainty and sensitivity analyses of a two-parameter impedance prediction model," 14th AIAA/CEAS Aeroacoustics Conference (29th AIAA Aeroacoustics Conference), 2008, p. 2928.

[5] Brown, M., Jones, M., and Watson, W., "Uncertainty analysis of the grazing flow impedance tube," 18th AIAA/CEAS Aeroacoustics Conference (33rd AIAA Aeroacoustics Conference), 2012, p. 2296.

[6] Zhou, L., and Bodén, H., "A systematic uncertainty analysis for liner impedance eduction technology," Journal of sound and vibration, Vol. 356, 2015, pp. 86-99.

[7] Nark, D. M., Jones, M. G., and Piot, E., "Assessment of axial wave number and mean flow uncertainty on acoustic liner impedance eduction,” 2018 AIAA/CEAS Aeroacoustics Conference, 2018, p. 3444.

[8] Bruneau, M., Manuel d'acoustique fondamentale, Hermes, 1998.

[9] Lighthill, M. J., “On sound generated aerodynamically. I. General theory,” Proceedings of the Royal Society of London A: Mathematical, Physical and Engineering Sciences, Vol. 211, The Royal Society, 1952, pp. 564-587.

[10] Landau, L. D., and Lifshitz, E., Fluid Mechanics: Vol 6, Elsevier, 2013.

[11] Hirsch, C., Numerical Computation of Internal and External Flows, Fundamentals of Numerical Discretization, Numerical Computation of Internal and External Flows, Wiley, 1991.

[12] Myers, M., "On the acoustic boundary condition in the presence of flow," Journal of Sound and Vibration, Vol. 71, No. 3, 1980, pp. 429-434.

[13] Lidoine, S., "Approches théoriques du problème du rayonnement acoustique par une entrée d'air de turboréacteur: Comparaisons entre différentes méthodes analytiques et numériques," Ph.D. thesis, Ecully, Ecole centrale de Lyon, 2002.

[14] Brambley, E. J., "Well-posed boundary condition for acoustic liners in straight ducts with flow," AIAA journal, Vol. 49, No. 6, 2011, pp. 1272-1282. 
[15] Guess, A., "Calculation of perforated plate liner parameters from specified acoustic resistance and reactance," Journal of Sound and Vibration, Vol. 40, No. 1, 1975, pp. 119-137.

[16] Hirschberg, A., and Rienstra, S. W., "An introduction to aeroacoustics," Eindhoven university of technology, 2004.

[17] Pierce, A. D., and Beyer, R. T., “Acoustics: An Introduction to Its Physical Principles and Applications. 1989 Edition,” , 1990.

[18] Soize, C., "A nonparametric model of random uncertainties for reduced matrix models in structural dynamics," Probabilistic engineering mechanics, Vol. 15, No. 3, 2000, pp. 277-294.

[19] Technologies, F. F., “Actran 12 User's Guide,”, 2011.

[20] Van Den Nieuwenhof, B., Detandt, Y., Lielens, G., Rosseel, E., Soize, C., Dangla, V., Kassem, M., and Mosson, A., "Optimal Design of the Acoustic Treatments Damping the Noise Radiated by a Turbo-Fan Engine," 23rd AIAA/CEAS Aeroacoustics Conference, 2017, p. 4035.

[21] Van Antwerpen, B., Detandt, Y., Copiello, D., Rosseel, E., and Gaudry, E., "Performance improvements and new solution strategies of Actran/TM for nacelle simulations," 20th AIAA/CEAS Aeroacoustics Conference, 2014, p. 2315.

[22] van Den Nieuwenhof, B., Detandt, Y., Lielens, G., Rosseel, E., Soize, C., Dangla, V., Kassem, M., and Mosson, A., "Optimal Design of the Acoustic Treatments Damping the Noise Radiated by a Turbo-Fan Engine," 23rd AIAA/CEAS Aeroacoustics Conference, 2017, p. 4035.

[23] Rienstra, S. W., "Fundamentals of duct acoustics," Von Karman Institute Lecture Notes, 2015.

[24] Ohayon, R., and Soize, C., Advanced computational vibroacoustics: reduced-order models and uncertainty quantification, Cambridge University Press, 2014.

[25] Mordillat, P., Dutrion, C., Petit, B., Rosseel, E., and Van den Nieuwenhof, B., "Robust Design of Acoustic Treatments for Powertrain Noise Radiation,” Tech. rep., SAE Technical Paper, 2018.

[26] Rubinstein, R. Y., and Kroese, D. P., Simulation and the Monte Carlo method, Vol. 10, John Wiley \& Sons, 2016.

[27] Shannon, C. E., "A mathematical theory of communication,” Bell system technical journal, Vol. 27, No. 3, 1948 , pp. $379-423$.

[28] Jaynes, E. T., "Information theory and statistical mechanics," Physical review, Vol. 106, No. 4, 1957, p. 620.

[29] Cover, T. M., and Thomas, J. A., "Elements of information theory 2nd edition (wiley series in telecommunications and signal processing)," 2006.

[30] Dickson, N., "ICAO noise standards," ICAO Symposium on Aviation and Climate Change. Retrieved from http://www. icao. int/Meetings/Green/Documents/day\% 201pdf/session, Vol. 202, 2013.

[31] Tyler, J. M., and Sofrin, T. G., “Axial flow compressor noise studies,” Tech. rep., SAE Technical Paper, 1962. 